\title{
Scalar blocks as gravitational Wilson networks
}

\section{Atanu Bhatta, Prashanth Raman and Nemani V. Suryanarayana}

Institute of Mathematical Sciences,

Taramani, Chennai 600 113, India

Homi Bhabha National Institute, Anushakti Nagar, Mumbai 400 085, India

E-mail: batanu@imsc.res.in, prashanthr@imsc.res.in, nemani@imsc.res.in

ABSTRACT: In this paper we continue to develop further our prescription [arXiv:1602.02962] to holographically compute the conformal partial waves of CFT correlation functions using the gravitational open Wilson network operators in the bulk. In particular, we demonstrate how to implement it to compute four-point scalar partial waves in general dimension. In the process we introduce the concept of OPE modules, that helps us simplify the computations. Our result for scalar partial waves is naturally given in terms of the Gegenbauer polynomials. We also provide a simpler proof of a previously known recursion relation for the even dimensional CFT partial waves, which naturally leads us to an odd dimensional counterpart.

KeYwords: AdS-CFT Correspondence, Conformal Field Theory, Classical Theories of Gravity, Conformal and W Symmetry

ARXIV EPRINT: 1806.05475 


\section{Contents}

1 Introduction 1

2 Scalar OWN in general dimensions $\quad 4$

2.1 Collecting the ingredients 4

2.2 Processing the ingredients $\quad 7$

$\begin{array}{ll}2.3 & \text { Introducing OPE module } \\ & 13\end{array}$

$\begin{array}{ll}2.4 \text { Computing the 4-point CPW } & 14\end{array}$

3 Recovery of results in $d \leq 4 \quad 16$

4 Seed blocks and recursion relations $\quad 19$

5 Some odds and ends $\quad 20$

$\begin{array}{ll}5.1 \text { Complete } d=1 \text { analysis } & 20\end{array}$

$5.2 \mathrm{OWN} s$ in more general $A d S_{3}$ geometries $\quad 22$

$\begin{array}{lll}6 & \text { Discussion } & 23\end{array}$

$\begin{array}{lll}\text { A CGC required for scalar CPW } & 24\end{array}$

B Manipulation of the $d$-dimensional result $\quad 30$

C Details of CPW computation in $d=4 \quad 31$

\section{Introduction}

The correlation function of a set of primary operators in a $d$-dimensional CFT can be decomposed into its partial waves. For example, the correlation function of four scalar primary operators can be decomposed as

$$
\left\langle\mathcal{O}_{1}\left(x_{1}\right) \mathcal{O}_{2}\left(x_{2}\right) \mathcal{O}_{3}\left(x_{3}\right) \mathcal{O}_{4}\left(x_{4}\right)\right\rangle=\sum_{\mathcal{O}} C_{12 \mathcal{O}} C^{\mathcal{O}}{ }_{34} W_{\Delta, l}^{(d)}\left(\Delta_{i}, x_{i}\right)
$$

where $C_{12 \mathcal{O}}$ are the OPE coefficients and the partial wave $W_{\Delta, l}^{(d)}\left(x_{i}\right)$ is

$$
W_{\Delta, l}^{(d)}\left(\Delta_{i}, x_{i}\right)=\left(\frac{x_{24}^{2}}{x_{14}^{2}}\right)^{\frac{1}{2}\left(\Delta_{1}-\Delta_{2}\right)}\left(\frac{x_{14}^{2}}{x_{13}^{2}}\right)^{\frac{1}{2}\left(\Delta_{3}-\Delta_{4}\right)}\left(x_{12}^{2}\right)^{-\frac{1}{2}\left(\Delta_{1}+\Delta_{2}\right)}\left(x_{34}^{2}\right)^{-\frac{1}{2}\left(\Delta_{3}+\Delta_{4}\right)} G_{\Delta, l}(u, v)
$$

The pre-factor is determined by the conformal invariance and the function $G_{\Delta, l}(u, v)$ referred to as the conformal block - depends only on the conformally invariant crossratios $u, v$. A lot is known about these conformal partial waves/blocks. For instance, 
a general expression for conformal partial waves (CPW) of four-point scalar correlators is given in [1] (see also [2,3]). Written in terms of the complex coordinates $z, \bar{z}$ where $u=z \bar{z}$ and $v=(1-z)(1-\bar{z})$, closed form expressions are known for all even $d$ for scalar CPW $[1,4]$. Also closed form expressions for scalar conformal blocks for particular choice $z=\bar{z}$ are known for all dimensions $[5,6]$. Powerful recursion relations between blocks in even $d$ are found in [4]. A different choice of parametrising the cross-ratios through $z=x e^{i \theta}$ and $\bar{z}=x e^{-i \theta}$ was also advocated in $[1,7]$.

Since AdS/CFT provides a natural avenue to answer questions in $\mathrm{CFT}_{d}$ in terms of $A d S_{d+1}$ gravity (and vice versa) it is natural to ask how to compute the conformal partial waves of a given correlation function of primary operators in a CFT holographically. To achieve this two distinct prescriptions have been proposed so far in the literature:

1. Geodesic Witten diagrams [8]: this prescription is based on the second order EinsteinHilbert formulation of gravity in which the conformal partial waves are given by the so called geodesic Witten diagrams. This has been generalised further in [9-18].

2. Gravitational open Wilson networks [19,20]: this prescription is suitable for the first order Hilbert-Palatini formulation of the bulk theory in which the conformal partial waves are given by appropriate gravitational open Wilson networks (OWN). These are studied and generalised for 2d CFTs in [21-24].

In this paper we restrict ourselves to the second prescription, and provide further computational methods for its implementation in general dimensions. Before proceeding further let us review some essential aspects of this construction (see [19] for more details).

In the first-order Hilbert-Palatini formulation of $A d S_{d+1}$ gravity $[25,26]$ the basic fields are the vielbeins $e^{a}$ and the spin-connections $\omega^{a b}$. They are conveniently combined into a 1-form gauge field $A$ in the adjoint of the $s o(1, d+1)$ algebra as:

$$
A=\frac{1}{l} e^{a} M_{0 a}+\frac{1}{2} \omega^{a b} M_{a b}
$$

where $\left\{M_{0 a}, M_{a b}\right\}$ are the generators of $s o(1, d+1)$ with $a, b=1, \cdots, d+1$. In this theory we consider a set of gauge covariant Wilson Network operators. In particular,

- One starts with an open, directed and trivalent graph (such as in figure 1) whose every line (internal as well as external) carries a representation label of the (Euclidean) conformal algebra $s o(1, d+1)$.

- The representations of interest are those non-unitary infinite dimensional irreps which are obtained by appropriate Wick rotation of the corresponding UIR of the associated Lorentzian conformal algebra $s o(2, d)$ of the $\mathrm{CFT}_{d}$. Such an irrep can be labeled by $\left(\Delta ; l_{1}, \cdots, l_{[d / 2]}\right)$ where $\Delta$ is the conformal weight and $l_{i}$ label which irrep the primary transforms in, under the boundary rotation group $s o(d)$.

- Next, one associates an open Wilson line (OWL) operator

$$
W_{y}^{x}(R, C)=P \exp \left[\int_{y}^{x} A\right]
$$




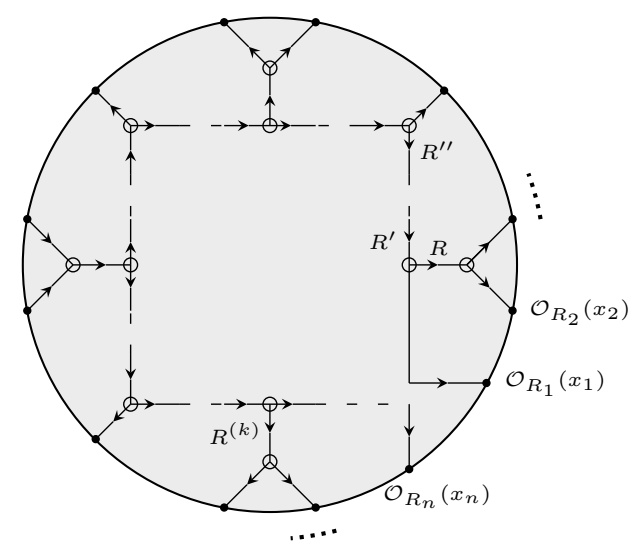

Figure 1. A typical directed trivalent Open Wilson Network.

for 1-form $A$ in (1.3) to the line labelled by the irrep $\mathrm{R}$, connecting the points $x$ and $y$ in the graph.

- At every trivalent vertex where three lines carrying representation labels $\left(R_{1}, R_{2}, R_{3}\right)$ join - one glues the corresponding OWLs with the appropriate Clebsch-Gordan coefficients to make the vertex gauge invariant.

- One projects each of the external lines onto Cap States [27-29] — a set of states in the conformal module $R$ labelling that leg that also provides a finite-dimensional irrep of the $s o(d+1)$ subalgebra whose generators are $M_{a b}$ used in (1.3).

- One evaluates these OWNs for the gauge connection $A$ that corresponds to the Euclidean Poincare $A d S_{d+1}$. Such a gauge connection has to satisfy the flatness condition

$$
F:=d A+A \wedge A=0 .
$$

- Finally one takes the external legs to the boundary and reads out the leading component of the OWN - and these compute the relevant conformal partial waves.

This leading component of the OWN satisfies the conformal Ward identities and conformal Casimir equations expected of the partial waves of a correlator of primaries that are inserted at the points on the boundary to which the end points of the external legs of the OWN approach.

In short, the basic ingredients needed to compute our OWNs are (i) Wilson lines, (ii) CG coefficient and (iii) the cap states. These were found for $d=2$ in [19] for the most general case. When the external legs were taken to the boundary the computation reduced to simple Feynman-like rules that require the knowledge of what we called legs (more precisely the conformal wave functions) and the CG coefficients. The explicit computations using these rules to find the global conformal blocks of correlators of primary operators (with any conformal dimension and spin) was demonstrated explicitly for $d=2$ in [19] (see also [20]). 
Even though the general prescription for computing the partial waves of correlators of any set of primaries (in arbitrary representations of the rotation group of the boundary theory) in general $\mathrm{CFT}_{d}$ using OWNs was laid down in [19], the actual computations in higher dimensions could not be carried through as some of the necessary ingredients were missing. In this work we would like to report some progress in this direction. In particular, we will demonstrate how to implement our prescription explicitly for the scalar CPW $W_{\Delta, 0}^{(d)}\left(\Delta_{i}, x_{i}\right)$ in any $\mathrm{CFT}_{d}$. Our results include a simplification of the computation of OWNs using the concept of OPE modules - which are close analogues of the OPE blocks that were studied in the literature [30,31]. With this simplification we compute the scalar 4-point blocks in general dimension and show that our prescription reproduces the known answers [1]. Remarkably, our results are naturally given in Gegenbauer polynomial basis [1, 7]. Further, we show that there is a non-trivial recursion relation that emerges from our prescription which relates the scalar blocks in $d+2$ dimensions to those of $d$ dimensions. This relation reproduces the one in [4] in the context of even $d$, and provides an analogue for the odd $d$ cases.

The rest of the paper is organised as follows: the section 2 contains the construction of the modules and the conformal wave functions required for the computation of scalar blocks. We also introduce the concept of OPE module here and use it to carry out the computation of the 4-point scalar blocks in general dimensions. The section 3 contains details of how our answers match with several known results in $d \leq 4$. In section 4 we derive recursion relations between different dimensions. In section 5 we include a couple of generalisations: most general bulk analysis in $d=1$, more general bulk geometries in $d=2$. We provide a discussion of our results and open questions in section 6 . The appendices contain some relevant mathematical results used in the text.

\section{Scalar OWN in general dimensions}

In this section we would like to provide details on how to explicitly compute the OWN $s$ in $A d S_{d+1}$ spaces, with all lines (both external and internal) carrying scalar representations.

\subsection{Collecting the ingredients}

As has been alluded to in the introduction the basic ingredients are Wilson lines, cap states and CG coefficients. We start with collecting these ingredients first.

Wilson lines. We will be evaluating the OWN in the background of the Euclidean $A d S_{d+1}$ geometry with $\mathbb{R}^{d}$ boundary (i.e, Poincare $A d S_{d+1}$ ) with the metric:

$$
l^{-2} d s_{A d S_{d+1}}^{2}=d \rho^{2}+e^{2 \rho} d x^{i} d x^{i} .
$$

For this, working with the frame:

$$
e^{i}=l e^{\rho} d x^{i}, i=1, \cdots, d, \quad e^{d+1}=l d \rho
$$

the Wilson line reduces to

$$
W_{y}^{x}(R, C)=P \exp \left[\int_{y}^{x} A\right]=g(x) g^{-1}(y)
$$


as was shown in [19], with

$$
g(x)=e^{-\rho M_{0, d+1}} e^{-x_{a}\left(M_{0, a}+M_{a, d+1}\right)} g_{0},
$$

where the algebra generators are taken in the representation $R$ of $s o(1, d+1)$. Using the standard identification of $s o(1, d+1)$ generators as the conformal generators of $\mathbb{R}^{d}$ :

$$
D=-M_{0, d+1}, \quad P_{\alpha}=M_{0 \alpha}+M_{\alpha, d+1}, \quad K_{\alpha}=-M_{0 \alpha}+M_{\alpha, d+1}, \quad \text { and } \quad M_{\alpha \beta}
$$

where $\alpha, \beta=1, \cdots, d$, the coset element $g(x)$ reads:

$$
g(x)=e^{\rho D} e^{-x^{a} P_{a}} g_{0}
$$

This gives us the Wilson lines.

The scalar caps. To project the external legs of the OWN operator we seek states, in the representation space $R$ carried by that external leg, that transforms in a (finite dimensional) irrep of the subalgebra $s o(d+1)$ with generators $\left\{M_{\alpha \beta}, M_{\alpha, d+1}\right\}$ [29]. In particular, for the scalar cap this finite dimensional representation is the trivial one, that is, annihilated by $\left\{M_{\alpha \beta}, M_{\alpha, d+1}\right\}$. Let us now construct these states.

In terms of the generators in $(2.5)$ the $s o(1, d+1)$ algebra reads

$$
\begin{aligned}
{\left[M_{\alpha \beta}, P_{\gamma}\right] } & =-\left(\delta_{\alpha \gamma} P_{\beta}-\delta_{\beta \gamma} P_{\alpha}\right), \quad\left[M_{\alpha \beta}, K_{\gamma}\right]=-\left(\delta_{\alpha \gamma} K_{\beta}-\delta_{\beta \gamma} K_{\alpha}\right), \\
{\left[P_{\alpha}, K_{\beta}\right] } & =-2 M_{\alpha \beta}-2 \delta_{\alpha \beta} D, \quad\left[D, P_{\alpha}\right]=P_{\alpha}, \quad\left[D, K_{\alpha}\right]=-K_{\alpha}, \\
{\left[M_{\alpha \beta}, M_{\gamma \delta}\right] } & =\delta_{\alpha \delta} M_{\beta \gamma}+\delta_{\beta \gamma} M_{\alpha \delta}-\delta_{\alpha \gamma} M_{\beta \delta}-\delta_{\beta \delta} M_{\alpha \gamma} .
\end{aligned}
$$

We work with irreps $R$ of $s o(1, d+1)$ that become UIR of $s o(2, d)$ obtained by a Wick rotation. This implies the following reality conditions

$$
M_{0, d+1}^{\dagger}=M_{0, d+1}, \quad M_{0 \alpha}^{\dagger}=-M_{0, \alpha}, \quad M_{\alpha, d+1}^{\dagger}=M_{\alpha, d+1}, \quad M_{\alpha \beta}^{\dagger}=-M_{\alpha \beta} .
$$

In terms of the generators in (2.5) these mean:

$$
D^{\dagger}=D, \quad P_{\alpha}^{\dagger}=K_{\alpha}, \quad M_{\alpha \beta}^{\dagger}=-M_{\alpha \beta}
$$

Then the scalar cap state $|\Delta\rangle\rangle$ is defined to be a state in the scalar module $\left(\Delta, l_{i}=0\right)$ that satisfies the conditions:

$$
\left.\left.M_{\alpha \beta}|\Delta\rangle\right\rangle=\left(P_{\alpha}+K_{\alpha}\right)|\Delta\rangle\right\rangle=0 .
$$

We can construct it as a linear combination of states in the module over the scalar primary (lowest weight) state $|\Delta\rangle$ which satisfies

$$
D|\Delta\rangle=\Delta|\Delta\rangle, \quad M_{\alpha \beta}|\Delta\rangle=K_{\alpha}|\Delta\rangle=0 .
$$

Rest of the basis states of the module take the form $\left|\Delta, k_{i}\right\rangle=\mathcal{N}_{\vec{k}} P_{1}^{k_{1}} \cdots P_{d}^{k_{d}}|\Delta\rangle$. The solution to the scalar cap state equation (2.11) was provided first in [29] (see also [27, 28] 
for $d=2$ case). We rederive it here for completeness. For this note that the cap state has to be a singlet under $s o(d)$ and therefore can only depend on $P_{\alpha} P^{\alpha}$. So write

$$
|\Delta\rangle\rangle=\sum_{n=0}^{\infty} C_{n}(\Delta, d)\left(P_{\alpha} P^{\alpha}\right)^{n}|\Delta\rangle,
$$

and impose $\left.\left(P_{\alpha}+K_{\alpha}\right)|\Delta\rangle\right\rangle=0$ to determine the coefficients $C_{n}$. Carrying out this straightforward exercise gives

$$
C_{n}(\Delta, d)=\frac{(-1)^{n}}{2^{2 n} n !(\Delta-\mu)_{n}}
$$

With these (2.12) can be seen to be equivalent to the one in [29] using the definition of the Bessel function of first kind $J_{\alpha}(x)$. We will need the dual (conjugate under (2.9)) of this cap state which is given by:

$$
\left\langle\langle\Delta|=\sum_{n=0}^{\infty} C_{n}(\Delta, d)\langle\Delta|\left(K_{\alpha} K^{\alpha}\right)^{n}\right.
$$

with the same $C_{n}$ as in $(2.13) .^{1}$

In fact one can obtain more general cap states. For instance, in the case of $d=2$, we [19] provided expressions for cap states in the module over the primary state $|h, \bar{h}\rangle$ that transform under $(j, m)$ representation of $s o(3)$ algebra. In other dimensions one should seek caps that transform under arbitrary finite dimensional irreps of $s o(d+1)$ - to be used in computing the OWN $s$ with primaries that are not just scalars (see (6.1) for the vector cap state - provided for illustration). We however will not pursue this further here.

CG coefficients. The last ingredient in the computation of the OWN expectation values is the Clebsch-Gordan coefficients (CGC) of the gauge algebra $s o(1, d+1)$. Some of these are known - see for instance [33]. Those are however not in a form that lends itself readily to our purposes. So here we propose a method to derive them using the 3-point functions.

For this first recall that the CG coefficients are defined as the invariant tensors in the product of three representations. That is, the CGC that appear in the tensor product decomposition $R_{1} \otimes R_{2} \rightarrow R_{3}$ satisfy:

$$
R_{1}[g(x)]_{\mathbf{m}_{1} \mathbf{m}_{1}^{\prime}} R_{2}[g(x)]_{\mathbf{m}_{2} \mathbf{m}_{2}^{\prime}} C_{\mathbf{m}_{1}^{\prime}, \mathbf{m}_{2}^{\prime} ; \mathbf{m}_{3}^{\prime}}^{R_{1}, R_{3} ; R_{3}} R_{3}\left[g(x)^{-1}\right]_{\mathbf{m}_{3}^{\prime} \mathbf{m}_{3}}=C_{\mathbf{m}_{1}, \mathbf{m}_{2} ; \mathbf{m}_{3}}^{R_{1}, R_{2} ; R_{3}}
$$

where $R_{i}[g(x)]_{\mathbf{m}_{i} \mathbf{m}_{i}^{\prime}}$ is used to denote the matrix elements of $g(x)$ in the representation $R_{i}$, whose basis elements are collectively labelled by $\mathbf{m}_{i}$. In terms of the algebra elements $M_{A B}$ with $A, B=0,1, \cdots, d+1$, this eq. (2.15) reads:

$$
R_{1}\left[M_{A B}\right]_{\mathbf{m}_{1} \mathbf{m}_{1}^{\prime}} C_{\mathbf{m}_{1}^{\prime}, \mathbf{m}_{2} ; \mathbf{m}_{3}}^{R_{1}, R_{2} R_{3}}+R_{2}\left[M_{A B}\right]_{\mathbf{m}_{2} \mathbf{m}_{2}^{\prime}} C_{\mathbf{m}_{1}, \mathbf{m}_{2}^{\prime} ; \mathbf{m}_{3}}^{R_{1}, R_{2} ; R_{3}}=C_{\mathbf{m}_{1}, \mathbf{m}_{2} ; \mathbf{m}_{3}^{\prime}}^{R_{1}, R_{3} ; R_{3}} R_{3}\left[M_{A B}\right]_{\mathbf{m}_{3}^{\prime} \mathbf{m}_{3}}
$$

which is the recursion relation that determines the CGC. Now we argue that this is equivalent to the conformal Ward identity of the 3-point function of primary operators corresponding to the irreps $\left(R_{1}, R_{2}, R_{3}\right)$. The prescription of [19] for the 3-point function of

\footnotetext{
${ }^{1}$ This scalar cap in the $d=2$ case can be seen to be equivalent to that with $h=\bar{h}$ cap used in [19] (see also [28] and more recently [32] for a different perspective).
} 


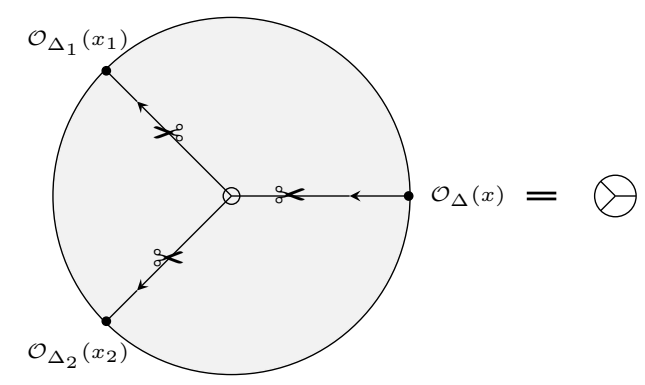

Figure 2. CG coefficients.

scalar primaries is to extract the leading term, i.e, the coefficient of $e^{-\rho\left(\Delta_{1}+\Delta_{2}+\Delta_{3}\right)}$ term — in the boundary limit of

$$
\left\langle\left\langle\Delta_{1}\left|g\left(x_{1}\right)\right| \Delta_{1}, \mathbf{m}_{1}\right\rangle\left\langle\left\langle\Delta_{2}\left|g\left(x_{2}\right)\right| \Delta_{2}, \mathbf{m}_{2}\right\rangle C_{\mathbf{m}_{1}, \mathbf{m}_{2} ; \mathbf{m}_{3}}^{\Delta_{1}, \Delta_{2} ; \Delta_{3}}\left\langle\Delta_{3}, \mathbf{m}_{3}\left|g^{-1}\left(x_{3}\right)\right| \Delta_{3}\right\rangle\right\rangle\right.
$$

We now show that this quantity satisfies the conformal Ward identity. To see this we note the following identities [19]:

$$
\begin{aligned}
g(x) M_{A B} & =l_{A B}^{\mu}(x) \partial_{\mu} g(x)+\frac{1}{2} M_{b c} g(x)\left[\omega_{\mu}^{b c}(x) l_{A B}^{\mu}(x)+(R[g(x)])_{A B}^{b c}{ }_{A B}\right. \\
M_{A B} g^{-1}(x) & =-l_{A B}^{\mu}(x) \partial_{\mu} g^{-1}(x)+\frac{1}{2}\left[\omega_{\mu}^{b c}(x) l_{A B}^{\mu}(x)+(R[g(x)])^{b c}{ }_{A B}\right] g^{-1}(x) M_{b c}
\end{aligned}
$$

where the $l_{A B}^{\mu}(x)$ are the components of the Killing vector of the background geometry (2.1) carrying the indices of the corresponding $s o(1, d+1)$ algebra generator $M_{A B} \in\left\{M_{0 a}, M_{a b}\right\}$ of the left hand side. Next we consider:

$$
\begin{aligned}
& \left\langle\left\langle\Delta_{1}\left|g\left(x_{1}\right) M_{A B}\right| \Delta_{1}, \mathbf{m}_{1}\right\rangle\left\langle\left\langle\Delta_{2}\left|g\left(x_{2}\right)\right| \Delta_{2}, \mathbf{m}_{2}\right\rangle C_{\mathbf{m}_{1}, \mathbf{m}_{2} ; \mathbf{m}_{3}}^{\Delta_{1}, \Delta_{2}}\left\langle\Delta_{3}, \mathbf{m}_{3}\left|g^{-1}\left(x_{3}\right)\right| \Delta_{3}\right\rangle\right\rangle\right. \\
& +\left\langle\left\langle\Delta_{1}\left|g\left(x_{1}\right)\right| \Delta_{1}, \mathbf{m}_{1}\right\rangle\left\langle\left\langle\Delta_{2}\left|g\left(x_{2}\right) M_{A B}\right| \Delta_{2}, \mathbf{m}_{2}\right\rangle C_{\mathbf{m}_{1}, \mathbf{m}_{2} ; \mathbf{m}_{3}}^{\Delta_{1}, \mathbf{m}_{3}}\left\langle\Delta_{3}, \mathbf{m}_{3}\left|g^{-1}\left(x_{3}\right)\right| \Delta_{3}\right\rangle\right\rangle\right. \\
& -\left\langle\left\langle\Delta_{1}\left|g\left(x_{1}\right)\right| \Delta_{1}, \mathbf{m}_{1}\right\rangle\left\langle\left\langle\Delta_{2}\left|g\left(x_{2}\right)\right| \Delta_{2}, \mathbf{m}_{2}\right\rangle C_{\mathbf{m}_{1}, \mathbf{m}_{2} ; \mathbf{m}_{3}}^{\Delta_{1} ; \Delta_{3}}\left\langle\Delta_{3}, \mathbf{m}_{3}\left|M_{A B} g^{-1}\left(x_{3}\right)\right| \Delta_{3}\right\rangle\right\rangle\right.
\end{aligned}
$$

which vanishes identically as a consequence of the recursion relation (2.16) for the CGC. On the other hand using the identities (2.18) above and the fact that the scalar cap is killed by $M_{a b}$ 's we see that the OWN for the 3-point function (2.17) is invariant under simultaneous transformation of the three bulk points $\left(x_{1}, x_{2}, x_{3}\right)$ under any $A d S_{d+1}$ isometry. This in turn implies the conformal Ward identity in the limit of the external points $x_{i}$ approaching the boundary. It is of course true that the Ward identity completely determines the coordinate dependence of the 3-point function. Therefore, the question of finding the CGC is translated into finding expressions for the quantities $\left\langle\langle\Delta|g(x)| \Delta, \mathbf{m}\rangle\right.$ and $\left.\left\langle\Delta, \mathbf{m}\left|g^{-1}(x)\right| \Delta\right\rangle\right\rangle$ in the large radius limit, and then amputating them from the corresponding 3-point function (figure 2). ${ }^{2}$

\subsection{Processing the ingredients}

To proceed further we need the explicit expressions for the in-going legs $\langle\langle\Delta|g(x)| \Delta, \mathbf{m}\rangle$ and the out-going legs $\left.\left\langle\Delta, \mathbf{m}\left|g^{-1}(x)\right| \Delta\right\rangle\right\rangle$ which are matrix elements of $g(x)$ and $g^{-1}(x)$ between

\footnotetext{
${ }^{2}$ Expressions of CGC for the scalar module obtained using this procedure can be found in appendix A.
} 
the cap states $|\Delta\rangle\rangle$ and normalised basis elements $|\Delta, \mathbf{m}\rangle$ of the scalar module. So we turn to finding a suitable orthonormal basis for the module over a scalar primary $|\Delta\rangle$ next.

Scalar module for $\boldsymbol{d} \geq \mathbf{2}$. The descendent states take the form $\left|\Delta,\left\{k_{1}, k_{2}, \ldots, k_{d}\right\}\right\rangle \sim$ $\prod_{i=1}^{d} P_{i}^{k_{i}}|\Delta\rangle$. These states are eigenstates of the dilatation operator $D$ with eigenvalue $\Delta+\sum_{i=1}^{d} k_{i}$. States with different eigenvalues of $D$ are orthogonal. The set of states with a given conformal weight form a reducible representation of the rotation algebra $s o(d)$ - which can be decomposed into a sum of irreps of $s o(d)$. Then states belonging to different irreps will also be orthogonal. Therefore, a more suitable basis to work with would be in terms of the hyperspherical harmonics of the boundary $s o(d)$ rotation algebra, $\left(P^{2}\right)^{s} M_{\mathbf{m}}^{l}(\mathbf{P})|\Delta\rangle$ where $\mathbf{m}$ denotes $\left(m_{d-2}, \cdots, m_{2}, m_{1}\right)$, whose conformal dimension is $\Delta+$ $l+2 s$. In the rest of the paper we follow the conventions of $[34,35]$ for hyperspherical functions. ${ }^{3}$ We define orthonormal states in this basis as follows ${ }^{4}$

$$
\begin{aligned}
\left(P^{2}\right)^{s} M_{\mathbf{m}}^{l}(\mathbf{P})|\Delta\rangle & :=A_{l, s}|\Delta ;\{l, \mathbf{m}, s\}\rangle \\
\langle\Delta|\left(K^{2}\right)^{s} M_{\mathbf{m}}^{l}(\mathbf{K}) & :=A_{l, s}^{*}\langle\Delta ;\{l, \mathbf{m}, s\}|
\end{aligned}
$$

with

$$
\left\langle\Delta ;\left\{l^{\prime}, \mathbf{m}^{\prime}, s^{\prime}\right\} \mid \Delta ;\{l, \mathbf{m}, s\}\right\rangle=\delta_{l l^{\prime}} \delta_{\mathbf{m m}^{\prime}} \delta_{s s^{\prime}}
$$

To find the normalisation $A_{l, s}$ let us start with the following observation

$$
\left\langle\Delta\left|e^{\mathbf{y} \cdot \mathbf{K}} e^{\mathbf{x} \cdot \mathbf{P}}\right| \Delta\right\rangle=\frac{1}{\left(1-2 \mathbf{x} \cdot \mathbf{y}+x^{2} y^{2}\right)^{\Delta}} .
$$

On the left hand side of the above identity we expand the plane waves $e^{\mathbf{x} \cdot \mathbf{P}}$ in terms of spherical waves: ${ }^{5}$

$$
e^{\mathbf{x} \cdot \mathbf{P}}=\sum_{l=0}^{\infty}(2 l+d-2)(d-4) ! ! j_{l}^{d}(x P) C_{l}^{\frac{d-2}{2}}\left(\frac{\mathbf{x} \cdot \mathbf{P}}{x P}\right)
$$

where $j_{l}^{d}(x)$ is the spherical Bessel function and $C_{l}^{\mu}(z)$ is the Gegenbauer polynomials as defined below

$$
C_{l}^{\frac{d-2}{2}}(x)=\frac{1}{(d-4) ! !} \sum_{k=0}^{[l / 2]}(-1)^{k} \frac{(2 l-2 k+d-4) ! !}{(2 k) ! !(l-2 k) ! !} x^{l-2 k}
$$

and

$$
j_{l}^{d}(x)=\sum_{s=0}^{\infty} \frac{(-1)^{s}(x)^{l+2 s}}{(2 s) ! !(d+2 l+2 s-2) ! !} .
$$

\footnotetext{
${ }^{3}$ It turns out that this choice is responsible for giving the CPWs as a sum over contributions of given spin $l$, namely the Gegenbauer polynomial basis.

${ }^{4}$ Note that the $s o(d)$ symmetry dictates that the normalisation of these states do not depend on $\mathbf{m}$.

${ }^{5}$ Even though this formal expansion looks odd as it apparently depends not only on $P$ whose square is $\mathbf{P} \cdot \mathbf{P}$, but also appears in the denominator of the argument of the Gegenbauer polynomial - we will shortly see that this is not a problem once interpreted correctly.
} 
One can also write Gegenbauer polynomials in terms of hyperspherical harmonics using the well known identity

$$
\sum_{\mathbf{m}} Y_{l ; \mathbf{m}}^{*}\left(\Omega_{x}\right) Y_{l ; \mathbf{m}}\left(\Omega_{y}\right)=\frac{\Gamma\left[\frac{d-2}{2}\right](2 l+d-2)}{4 \pi^{d / 2}} C_{l}^{\frac{d-2}{2}}\left(\frac{\vec{x} \cdot \vec{y}}{x y}\right)
$$

Substituting these into the (2.24) we get:

$$
e^{\mathbf{x} \cdot \mathbf{P}}=4 a \pi^{\frac{d}{2}} \sum_{l=0}^{\infty} \sum_{s=0}^{\infty} \frac{\left(x^{2}\right)^{s}}{2^{l+2 s} s ! \Gamma\left[l+s+\frac{d}{2}\right]} \sum_{\mathbf{m}} M_{\mathbf{m}}^{l *}(\mathbf{x}) M_{\mathbf{m}}^{l}(\mathbf{P})\left(P^{2}\right)^{s}
$$

where $M_{\mathbf{m}}^{l}(\mathbf{x})=x^{l} Y_{l ; \mathbf{m}}\left(\Omega_{x}\right)$ and

$$
a= \begin{cases}\frac{1}{2^{(d-2) / 2} \Gamma\left[\frac{d-2}{2}\right]}, & \text { if } d \text { is even } \\ \frac{\sqrt{\pi}}{2^{(d-1) / 2} \Gamma\left[\frac{d-2}{2}\right]}, & \text { if } d \text { is odd }\end{cases}
$$

Similarly

$$
e^{\mathbf{y} \cdot \mathbf{K}}=4 a \pi^{\frac{d}{2}} \sum_{l=0}^{\infty} \sum_{s=0}^{\infty} \frac{\left(y^{2}\right)^{s}}{2^{l+2 s} s ! \Gamma\left[l+s+\frac{d}{2}\right]} \sum_{\mathbf{m}} M_{\mathbf{m}}^{l *}(\mathbf{y}) M_{\mathbf{m}}^{l}(\mathbf{K})\left(K^{2}\right)^{s}
$$

Therefore the left hand side of (2.23) takes the following form

$$
\left\langle\Delta\left|e^{\mathbf{y} \cdot \mathbf{K}} e^{\mathbf{x} \cdot \mathbf{P}}\right| \Delta\right\rangle=\sum_{l=0}^{\infty} \sum_{s=0}^{\infty}\left(x^{2}\right)^{s}\left(y^{2}\right)^{s} \sum_{\mathbf{m}} M_{\mathbf{m}}^{l}(\mathbf{y}) M_{\mathbf{m}}^{l *}(\mathbf{x})\left|A_{l, s}\right|^{2}\left(\frac{4 a \pi^{\frac{d}{2}}}{s ! 2^{l+2 s} \Gamma(l+s+d / 2)}\right)^{2}
$$

Next we want to expand the r.h.s. of (2.23) in the same basis. For this we first write

$$
\frac{1}{\left(1-2 \mathbf{x} \cdot \mathbf{y}+x^{2} y^{2}\right)^{\Delta}}=\frac{1}{\left(1-2 \xi t+t^{2}\right)^{\Delta}}
$$

with $t=x y$ and $\xi=t^{-1} \mathbf{x} \cdot \mathbf{y}$. We would now like to expand this quantity in terms of Gegenbauer polynomials $C_{n}^{\mu}(x)$. Luckily this exercise was done in [36] which reads ${ }^{6}$

$$
\frac{1}{\left(1-2 \xi t+t^{2}\right)^{\Delta}}=\frac{\Gamma(\mu)}{\Gamma(\Delta)} \sum_{k=0}^{\infty} C_{k}^{\mu}(\xi) t^{k} \frac{\Gamma(\Delta+k)}{\Gamma(\mu+k)}{ }_{2} F_{1}\left(\Delta+k, \Delta-\mu ; \mu+k+1 ; t^{2}\right)
$$

However, we are interested in expanding the l.h.s. of (2.33) in $d$-dimensional hyperspherical harmonics in $\mathbf{x}$ which requires us to choose $\mu=(d-2) / 2$. Using the series representation of the hypergeometric function:

$$
{ }_{2} F_{1}\left(\Delta+k, \Delta-\mu ; \mu+k+1 ; t^{2}\right)=\frac{\Gamma(\mu+k+1)}{\Gamma(\Delta+k) \Gamma(\Delta-\mu)} \sum_{n=0}^{\infty} \frac{\Gamma(\Delta+k+n) \Gamma(\Delta-\mu+n)}{\Gamma(\mu+k+n+1)} \frac{t^{2 n}}{n !}
$$

\footnotetext{
${ }^{6}$ This is a remarkable generalisation of how the Gegenbauer Polynomials $C_{k}^{\mu}(x)$ are defined through its generating function when $\Delta=\mu$.
} 
and using the identity (2.27) we finally arrive at

$$
\begin{aligned}
\frac{1}{\left(1-2 \mathbf{x} \cdot \mathbf{y}+x^{2} y^{2}\right)^{\Delta}}= & \frac{4 \pi^{\frac{d}{2}}}{\Gamma(\Delta)} \sum_{l, s=0}^{\infty} \frac{\Gamma(\Delta+l+s) \Gamma\left(\Delta+s-\frac{d-2}{2}\right)}{\Gamma(l+s+d / 2) s !}\left(x^{2}\right)^{l+2 s}\left(y^{2}\right)^{l+2 s} \\
& \times \sum_{\mathbf{m}} M_{\mathbf{m}}^{l}(\mathbf{y}) M_{\mathbf{m}}^{l *}(\mathbf{x})
\end{aligned}
$$

Comparing (2.31) with (2.35), we get $^{7}$

$$
\left|A_{l, s}\right|^{2}=\frac{2^{2 l+4 s} \Gamma[l+s+d / 2] \Gamma[\Delta+l+s] \Gamma\left[\Delta+s-\frac{(d-2)}{2}\right] s !}{4 a^{2} \pi^{\frac{d}{2}} \Gamma[d / 2] \Gamma[\Delta] \Gamma\left[\Delta-\frac{(d-2)}{2}\right]}
$$

Having found an orthonormal basis for the scalar module we would like to now compute the legs (conformal wave functions) as described in the beginning of this section.

In-going legs. For this we start with $g(x)=e^{\rho D} e^{-\mathbf{x} \cdot \mathbf{P}}$. Then

$$
\begin{aligned}
\langle\langle\Delta|g(x)| \Delta ;\{l, \mathbf{m}, s\}\rangle & \\
= & \sum_{n=0}^{\infty}(-1)^{n} C_{n}\left\langle\Delta\left|\left(K^{2}\right)^{n} e^{\rho D} e^{-\mathbf{x} \cdot \mathbf{P}}\right| \Delta ;\{l, \mathbf{m}, s\}\right\rangle \\
= & \sum_{n=0}^{\infty} \frac{(-1)^{n} C_{n}}{A_{l, s}} A_{0, n}^{*}\left\langle\Delta,\{0,0, n\}\left|e^{\rho D} e^{-\mathbf{x} \cdot \mathbf{P}}\left(P^{2}\right)^{s} M_{\mathbf{m}}^{l}(\mathbf{P})\right| \Delta\right\rangle \\
= & \frac{4 a \pi^{\frac{d}{2}}}{A_{l, s}} \sum_{n=0}^{\infty}(-1)^{n} C_{n} A_{0, n}^{*} \sum_{l^{\prime}=0}^{\infty} \sum_{s^{\prime}=0}^{\infty} \frac{\left(x^{2}\right)^{s^{\prime}}}{s^{\prime} ! 2^{l^{\prime}+2 s^{\prime}} \Gamma\left(l^{\prime}+s^{\prime}+d / 2\right)} \sum_{\mathbf{m}^{\prime}} M_{\mathbf{m}^{\prime}}^{l^{\prime *}}(-\mathbf{x}) \\
& \times\left\langle\Delta,\{0,0, n\}\left|e^{\rho D}\left(P^{2}\right)^{s+s^{\prime}} M_{\mathbf{m}^{\prime}}^{l^{\prime}}(\mathbf{P}) M_{m}^{l}(\mathbf{P})\right| \Delta\right\rangle
\end{aligned}
$$

Now using the identity for the hyperspherical harmonics

$$
M_{\mathbf{m}}^{l}(\mathbf{P}) M_{\mathbf{m}^{\prime}}^{l^{\prime}}(\mathbf{P})=\sum_{L} \sum_{\mathbf{n}}\left[\begin{array}{ccc}
l & l^{\prime} & L \\
\mathbf{m} & \mathbf{m}^{\prime} & \mathbf{n}
\end{array}\right]\left(P^{2}\right)^{\frac{l+l^{\prime}-L}{2}} M_{\mathbf{n}}^{L}(\mathbf{P})
$$

where $\left[\begin{array}{ccc}l & l^{\prime} & L \\ \mathbf{m} & \mathbf{m}^{\prime} & \mathbf{n}\end{array}\right]$ is $s o(d)$ CG coefficients, we find

$$
\begin{aligned}
& \langle\langle\Delta|g(x)| \Delta ;\{l, \mathbf{m}, s\}\rangle \\
& =\frac{4 a \pi^{\frac{d}{2}}}{A_{l, s}} \sum_{n=0}^{\infty}(-1)^{n} C_{n} A_{0, n}^{*} \sum_{l^{\prime}=0}^{\infty} \sum_{s^{\prime}=0}^{\infty} \frac{\left(x^{2}\right)^{s^{\prime}}}{s^{\prime} ! 2^{l^{\prime}+2 s^{\prime}} \Gamma\left(l^{\prime}+s^{\prime}+d / 2\right)} \sum_{\mathbf{m}^{\prime}} M_{\mathbf{m}^{\prime}}^{l^{\prime} *}(-\mathbf{x}) e^{\rho\left(\Delta+l+l^{\prime}+2\left(s+s^{\prime}\right)\right)} \\
& \times \sum_{L} \sum_{\mathbf{n}}\left[\begin{array}{ccc}
l & l^{\prime} & L \\
\mathbf{m} & \mathbf{m}^{\prime} & \mathbf{n}
\end{array}\right]\left\langle\Delta ;\{0,0, n\}\left|\left(P^{2}\right)^{s+s^{\prime}+\left(l+l^{\prime}-L\right) / 2} M_{\mathbf{n}}^{L}(\mathbf{P})\right| \Delta\right\rangle \\
& =\frac{4 a \pi^{\frac{d}{2}}}{A_{l, s}} \sum_{n=0}^{\infty}(-1)^{n} C_{n} A_{0, n}^{*} \sum_{l^{\prime}=0}^{\infty} \sum_{s^{\prime}=0}^{\infty} \frac{\left(x^{2}\right)^{s^{\prime}}}{s^{\prime} ! 2^{l^{\prime}+2 s^{\prime}} \Gamma\left(l^{\prime}+s^{\prime}+d / 2\right)} \sum_{\mathbf{m}^{\prime}} M_{\mathbf{m}^{\prime}}^{l^{\prime} *}(-\mathbf{x}) e^{\rho\left(\Delta+l+l^{\prime}+2\left(s+s^{\prime}\right)\right)} \\
& \times \sum_{L} \sum_{\mathbf{n}}\left[\begin{array}{ccc}
l & l^{\prime} & L \\
\mathbf{m} & \mathbf{m}^{\prime} & \mathbf{n}
\end{array}\right] A_{L, s+s^{\prime}+\frac{l+l^{\prime}-L}{2}} \delta_{L 0} \delta_{\mathbf{n} 0} \delta_{n\left(s+s^{\prime}+\frac{l+l^{\prime}-L}{2}\right)}
\end{aligned}
$$

\footnotetext{
${ }^{7}$ While this work was in progress [37] appeared where the same result was obtained in a different context.
} 
Carrying out the summation over $L$ and $\mathbf{n}$ we find

$$
\begin{aligned}
& \langle\langle\Delta|g(x)| \Delta ;\{l, \mathbf{m}, s\}\rangle \\
& =\frac{4 a \pi^{\frac{d}{2}}}{A_{l, s}} \sum_{n=0}^{\infty}(-1)^{n} C_{n} A_{0, n}^{*} \sum_{l^{\prime}=0}^{\infty} \sum_{s^{\prime}=0}^{\infty} \frac{\left(x^{2}\right)^{s^{\prime}}}{s^{\prime} ! 2^{l^{\prime}+2 s^{\prime}} \Gamma\left(l^{\prime}+s^{\prime}+d / 2\right)} M_{\mathbf{m}}^{l}(-\mathbf{x}) e^{\rho\left(\Delta+l+l^{\prime}+2\left(s+s^{\prime}\right)\right)} \\
& \times \delta_{l l^{\prime}} A_{0, s+s^{\prime}+\frac{l+l^{\prime}}{2}} \delta_{n\left(s+s^{\prime}+\frac{l+l^{\prime}}{2}\right)}(2.38)
\end{aligned}
$$

where we have used

$$
\sum_{\mathbf{m}^{\prime}}\left[\begin{array}{ccc}
l & l^{\prime} & 0 \\
\mathbf{m} & \mathbf{m}^{\prime} & 0
\end{array}\right] M_{\mathbf{m}^{\prime}}^{l^{\prime} *}(\mathbf{x})=\delta_{l l^{\prime}} M_{\mathbf{m}}^{l}(\mathbf{x})
$$

Therefore

$$
\begin{aligned}
& \langle\langle\Delta|g(x)| \Delta ;\{l, \mathbf{m}, s\}\rangle \\
& =\frac{4 a \pi^{\frac{d}{2}}}{A_{l, s}} \sum_{n=0}^{\infty}(-1)^{n} C_{n} A_{0, n}^{*} \sum_{s^{\prime}=0}^{\infty} \frac{\left(x^{2}\right)^{s^{\prime}}}{s^{\prime} ! 2^{l+2 s^{\prime}} \Gamma\left(l+s^{\prime}+d / 2\right)} \\
& \times M_{\mathbf{m}}^{l}(-\mathbf{x}) e^{\rho\left(\Delta+2\left(l+s+s^{\prime}\right)\right)} A_{0, s+s^{\prime}+l} \delta_{n\left(s+s^{\prime}+l\right)} \\
& =e^{\rho \Delta} \frac{4 a \pi^{\frac{d}{2}}}{A_{l, s}}\left(x^{2}\right)^{-l-s} M_{\mathbf{m}}^{l}(-\mathbf{x}) \sum_{n=0}^{\infty}(-1)^{n} C_{n}\left|A_{0, n}\right|^{2} \frac{\left(e^{2 \rho} x^{2}\right)^{n}}{(n-s-l) ! 2^{2 n-2 s-l} \Gamma(n-s+d / 2)} \\
& =e^{-\rho \Delta} \frac{4 a \pi^{d / 2} 2^{l+2 s}}{A_{l, s}} \times M_{\mathbf{m}}^{l}(-\mathbf{x}) \times(-1)^{s+l}\left(e^{2 \rho}\right)^{\Delta+l+s}(l+d / 2)_{s}(\Delta)_{l+s} \\
& \times{ }_{2} F_{1}\left(\Delta+l+s, l+s+d / 2 ; l+d / 2 ;-e^{2 \rho} x^{2}\right)
\end{aligned}
$$

Now we want to take $\rho \rightarrow \infty$ limit. We rewrite the hypergeometric function in the above expression using the identity

$$
{ }_{2} F_{1}(a, b ; c ; z)=(1-z)_{2}^{-a} F_{1}\left(a, c-b ; c ; \frac{z}{z-1}\right)
$$

as

$$
\begin{aligned}
{ }_{2} F_{1}(\Delta+l+s & \left., l+s+d / 2 ; l+d / 2 ;-e^{2 \rho} x^{2}\right) \\
& =\left(1+e^{2 \rho} x^{2}\right)^{-\Delta-l-s}{ }_{2} F_{1}\left(\Delta+l+s,-s ; l+\frac{d}{2} ; \frac{e^{2 \rho} x^{2}}{1+e^{2 \rho} x^{2}}\right)
\end{aligned}
$$

In the $\rho \rightarrow \infty$ limit the argument of the hypergeometric function tends to unity. As the following identity holds

$$
{ }_{2} F_{1}(-n, b ; c ; 1)=\frac{(c-b)_{n}}{(c)_{n}}=\frac{\Gamma(c-b+n) \Gamma(c)}{\Gamma(c-b) \Gamma(c+n)},
$$

to the leading order in $e^{\rho}$ the in-going leg becomes

$$
\begin{aligned}
& \langle\langle\Delta|g(x)| \Delta ;\{l, \mathbf{m}, s\}\rangle \\
& \quad \rightarrow e^{-\rho \Delta} \frac{4 a \pi^{d / 2} 2^{l+2 s}}{A_{l, s}}(-1)^{s+l} M_{\mathbf{m}}^{l}(-\mathbf{x})\left(x^{2}\right)^{-\Delta-l-s}(\Delta)_{l+s}(d / 2-\Delta-s)_{s}+\cdots
\end{aligned}
$$


where dots are subleading terms in $\rho \rightarrow \infty$ limit. Finally we use $(-x)_{n}=(-1)^{n}(x-n+1)_{n}$ and $(-1)^{l} M_{\mathbf{m}}^{l}(\mathbf{x})=M_{\mathbf{m}}^{l}(-\mathbf{x})$ to get

$$
\lim _{\rho \rightarrow \infty} e^{\rho \Delta}\left\langle\langle\Delta|g(x)| \Delta ;\{l, \mathbf{m}, s\}\rangle=4 a \pi^{d / 2} \frac{2^{l+2 s}}{A_{l, s}}(\Delta)_{l+s}\left(\Delta-\frac{d-2}{2}\right)_{s}\left(x^{2}\right)^{-\Delta-l-s} M_{\mathbf{m}}^{l}(\mathbf{x})\right.
$$

Out-going legs. For this we start with $g^{-1}(y)=e^{\mathbf{y} \cdot \mathbf{P}} e^{-\rho D}$, and compute

$$
\begin{aligned}
\langle\Delta ; & \left.\left.\{l, \mathbf{m}, s\}\left|g^{-1}(y)\right| \Delta\right\rangle\right\rangle \\
& =\sum_{n=0}^{\infty}(-1)^{n} C_{n} e^{-\rho(\Delta+2 n)}\left\langle\Delta ;\{l, \mathbf{m}, s\}\left|e^{\mathbf{y} \cdot \mathbf{P}}\left(P^{2}\right)^{n}\right| \Delta\right\rangle \\
& =4 a \pi^{d / 2} \sum_{n=0}^{\infty}(-1)^{n} C_{n} e^{-\rho(\Delta+2 n)} \sum_{l^{\prime}=0}^{\infty} \sum_{s^{\prime}=0}^{\infty} \frac{\left(y^{2}\right)^{s^{\prime}}}{s^{\prime} ! 2^{l^{\prime}+2 s^{\prime}} \Gamma\left(l^{\prime}+s^{\prime}+d / 2\right)} \sum_{\mathbf{m}^{\prime}} M_{\mathbf{m}^{\prime}}^{l^{\prime} *}(\mathbf{y}) \\
& =4 a \pi^{d / 2} \sum_{n=0}^{\infty}(-1)^{n} C_{n} A_{l, s} e^{-\rho(\Delta+2 n)} \frac{\left(A_{l^{\prime}, s^{\prime}+n} \delta_{l l^{\prime}}\right.}{\delta_{\mathbf{m m}^{\prime}}} \delta_{s\left(s^{\prime}+n\right)} \\
& =e^{-\rho \Delta} \frac{4 a \pi^{d / 2}}{2^{l+2 s}} A_{l, s} \sum_{n=0}^{\infty}(-1)^{n} C_{n} e^{-2 n \rho} \frac{\left(y^{2}\right)^{s-n}}{(s-n) ! \Gamma(l+s-n) \Gamma(l+s-n+d / 2)} M_{\mathbf{m}}^{l *}(\mathbf{y})
\end{aligned}
$$

As $\rho \rightarrow \infty$, to the leading order only the $n=0$ term contributes, so that we have the result

$$
\left.\lim _{\rho \rightarrow \infty} e^{\rho \Delta}\left\langle\Delta ;\{l, \mathbf{m}, s\}\left|g^{-1}(y)\right| \Delta\right\rangle\right\rangle=\frac{4 a \pi^{d / 2}}{2^{l+2 s}} A_{l, s} \frac{\left(y^{2}\right)^{s}}{(s) ! \Gamma(l+s+d / 2)} M_{\mathbf{m}}^{l *}(\mathbf{y})
$$

The results of these rather lengthy, albeit straightforward exercises are (2.44), (2.46). These two sets of functions (2.44) and (2.46) provide a representation and its conjugate representation respectively of the conformal algebra $s o(1, d+1)$, on which the conformal generators $\left\{D, M_{\alpha \beta}, P_{\alpha}, K_{\alpha}\right\}$ act through their differential operator representations on scalar primaries with dimension $\Delta$. One can use these to derive matrix representations of the conformal generators and therefore, can be more appropriately called the conformal wave functions.

Finally let us quickly carry out a check on our conformal wave functions, namely, that when they are used in our OWN prescription they have to reproduce the appropriate two-point function for the scalar primaries. According to our prescription the two-point function can be obtained as

$$
\begin{aligned}
\left\langle\mathcal{O}_{\Delta}(\mathbf{x}) \mathcal{O}_{\Delta}(\mathbf{y})\right\rangle & =\lim _{\rho \rightarrow \infty} e^{2 \Delta \rho}\left\langle\left\langle\Delta\left|g(x) g^{-1}(y)\right| \Delta\right\rangle\right\rangle \\
& =\lim _{\rho \rightarrow \infty} e^{2 \Delta \rho} \sum_{l=0}^{\infty} \sum_{s=0}^{\infty} \sum_{\mathbf{m}}\left\langle\langle\Delta|g(x)| \Delta ;\{l, \mathbf{m}, s\}\rangle\left\langle\Delta ;\{l, \mathbf{m}, s\}\left|g^{-1}(y)\right| \Delta\right\rangle\right\rangle
\end{aligned}
$$

As $\rho \rightarrow \infty$ the above diagram evaluates to

$$
\begin{aligned}
& \sum_{l=0}^{\infty} \sum_{s=0}^{\infty} \sum_{m=-l}^{l}\left\langle\langle\Delta|g(x)| \Delta ;\{l, m, s\}\rangle\left\langle\Delta ;\{l, m, s\}\left|g^{-1}(y)\right| \Delta\right\rangle\right\rangle \\
& =e^{-2 \Delta \rho}\left(x^{2}\right)^{-\Delta}\left(4 a \pi^{d / 2}\right)^{2} \sum_{l=0}^{\infty} \sum_{s=0}^{\infty} \frac{(\Delta)_{l+s}\left(\Delta-\frac{d-2}{2}\right)_{s}}{\Gamma(l+s+d / 2) s !}\left(\frac{y}{x}\right)^{2 s}\left(x^{2}\right)^{-l} \sum_{\mathbf{m}} M_{\mathbf{m}}^{l}(\mathbf{x}) M_{\mathbf{m}}^{l *}(\mathbf{y})
\end{aligned}
$$




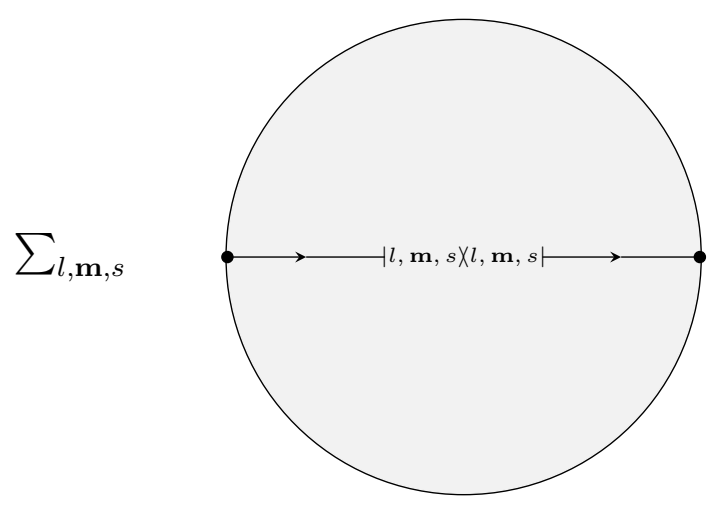

Figure 3. 2-point function.

Finally using (2.27) and comparing with (2.33) we obtain

$$
\begin{aligned}
\left\langle\mathcal{O}_{\Delta}(\mathbf{x}) \mathcal{O}_{\Delta}(\mathbf{y})\right\rangle & =4 a^{2} \pi^{d / 2}\left(x^{2}\right)^{-\Delta}\left(1-2 \frac{\mathbf{x} \cdot \mathbf{y}}{x^{2}}+\frac{y^{2}}{x^{2}}\right)^{-\Delta} \\
& =4 a^{2} \pi^{d / 2}|\mathbf{x}-\mathbf{y}|^{-2 \Delta}
\end{aligned}
$$

This is the expected result for two-point function (up to an overall constant factor - which can be gotten rid of by multiplying the cap states by appropriate overall factors).

\subsection{Introducing OPE module}

Finally we need to amputate the legs (2.44), (2.46) we have found in the previous subsection from the correlation function of three scalar primaries to find the CGC we need. The explicit expressions adapted to our method are given in appendix A. However, to compute, for example, the 4-point conformal partial waves we need CGC $s$ that are already connected to two legs at a time - which is obtained easily by starting with an appropriate 3-point function and amputating only one leg. This object depends on the boundary coordinates where two of the primaries are inserted, and carries labels of basis vectors of the module of the third primary. This is a close cousin of the so called OPE block [30,31], which we call the OPE module.

These OPE modules can be characterised by two types of identities. To spell them out let us label the representations of the conformal algebra $\operatorname{so}(1, d+1)$ of interest by $(\Delta, 1)$ where $\Delta$ is the conformal dimension and $\mathbf{l}$ represents all the independent Casimirs of the representation. States in such a representation $R$ can be labelled by $(\Delta, \mathbf{l} ; \mathbf{m}, s)$ where $\mathbf{m}$ is again a collective index of magnetic quantum numbers. It turns out there are two types of these OPE modules which we denote by $\mathcal{B}_{\left(\Delta_{3}, \mathbf{l}_{3} ; \mathbf{m}_{3}, s_{3}\right)}^{\left(\Delta_{1}, \mathbf{l}_{1} ; \mathbf{l}_{1} ; \mathbf{x}_{2}\right)}$ and $\mathcal{B}_{\left(\Delta_{1}, \mathbf{l}_{1} ; \mathbf{x}_{1}\right),\left(\Delta_{2}, \mathbf{l}_{2} ; \mathbf{x}_{2}\right)}^{\left(\Delta_{\Delta_{3}} \mathbf{l}_{3} \mathbf{m}_{3}, s_{3}\right)}$. Then these OPE modules are supposed to satisfy the Ward identities:

$$
\begin{aligned}
& \left(\mathcal{L}_{x_{1}}\left[M_{A B}\right]+\mathcal{L}_{x_{2}}\left[M_{A B}\right]\right) \mathcal{B}_{(\Delta, \mathbf{l} ; \mathbf{m}, s)}^{\left(\Delta_{1} \mathbf{l}_{1} ; \mathbf{x}_{1}\right),\left(\Delta_{2}, \mathbf{l}_{2} ; \mathbf{x}_{2}\right)}=\mathcal{M}_{(\Delta, \mathbf{l} ; \mathbf{m}, s)}^{\left(\Delta, \mathbf{l} ; \mathbf{m}^{\prime}, s^{\prime}\right)}\left[M_{A B}\right] \mathcal{B}_{\left(\Delta, \mathbf{l} ; \mathbf{m}^{\prime}, s^{\prime}\right)}^{\left(\Delta_{1} \mathbf{l}_{1} ; \mathbf{x}_{1}\right),\left(\Delta_{2}, \mathbf{l}_{2} ; \mathbf{x}_{2}\right)} \\
& \left(\mathcal{L}_{x_{1}}\left[M_{A B}\right]+\mathcal{L}_{x_{2}}\left[M_{A B}\right]\right) \mathcal{B}_{\left(\Delta_{1}, \mathbf{l}_{1} ; \mathbf{x}_{1}\right),\left(\Delta_{2}, \mathbf{l}_{2} ; \mathbf{x}_{2}\right)}^{(\Delta, \mathbf{l} ; \mathbf{m})}=-\mathcal{B}_{\left(\Delta_{1}, \mathbf{l}_{1} ; \mathbf{x}_{1}\right),\left(\Delta_{2}, \mathbf{l}_{2} ; \mathbf{x}_{2}\right)}^{\left(\Delta, \mathbf{l} ; \mathbf{x}^{\prime},{ }^{\prime}\right)} \\
& \times \mathcal{M}_{\left(\Delta, \mathbf{l} ; \mathbf{m}^{\prime}, s^{\prime}\right)}^{(\Delta, \mathbf{l} ; \mathbf{m}, s)}\left[M_{A B}\right]
\end{aligned}
$$




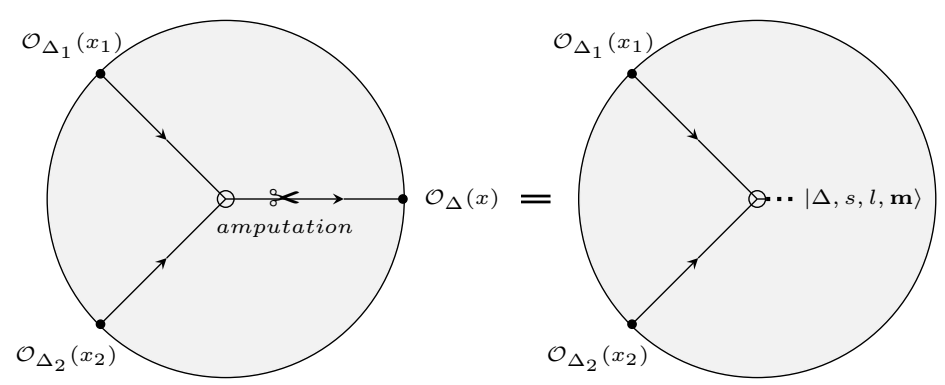

Figure 4. OPE module from 3-point function.

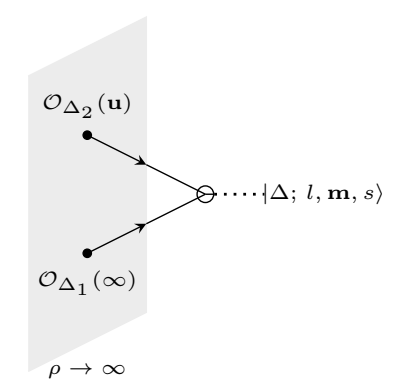

Figure 5. An OPE module.

where we denote the differential operator representation and the matrix representation of the conformal generator $M_{A B}$ by $\mathcal{L}\left[M_{A B}\right]$ and $\mathcal{M}\left[M_{A B}\right]$ respectively. From these identities it is very easy to see that both types of OPE modules satisfy the corresponding conformal Casimir equations.

For the scalar blocks of interest here, the two types of OPE modules can be obtained by amputating either an in-going (2.44), or an out-going leg (2.46) from the appropriate 3-point functions: $\left\langle\mathcal{O}_{\Delta_{1}}\left(x_{1}\right) \mathcal{O}_{\Delta_{2}}\left(x_{2}\right) \mathcal{O}_{\Delta}(x)\right\rangle,\left\langle\mathcal{O}_{\Delta}(x) \mathcal{O}_{\Delta_{3}}\left(x_{3}\right) \mathcal{O}_{\Delta_{4}}\left(x_{4}\right)\right\rangle$. See figure 4 for a pictorial representation of this procedure.

Finally the method to obtain the 4-point conformal partial wave using the OWN prescription reduces to taking two types of OPE modules defined above and contracting the module indices.

\subsection{Computing the 4-point CPW}

Having equipped ourselves with all the ingredients needed, we now turn to compute fourpoint conformal blocks for scalar primaries of conformal weights $\Delta_{i}$ for $i=1,2,3,4$. For simplicity we take the operator insertion points to be at $\mathbf{x}_{\mathbf{1}} \rightarrow \infty, \mathbf{x}_{\mathbf{2}} \rightarrow \mathbf{u}, \mathbf{x}_{\mathbf{3}} \rightarrow \mathbf{x}$ and $\mathbf{x}_{\mathbf{4}} \rightarrow \mathbf{0}$ with $\mathbf{u} \cdot \mathbf{u}=1$. As elucidated above this four-point conformal block can be computed using two specific OPE modules.

One of the OPE modules we need can be extracted from the three-point function, with the operator insertions at $(\infty, \mathbf{u}, \mathbf{y})$ by amputating the out-going leg anchored at the boundary-point $\mathbf{y}$. The corresponding OPE module is shown in the figure 5 below. The 


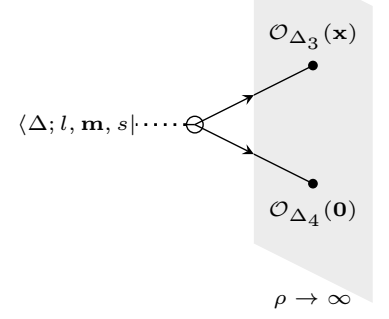

Figure 6. Another OPE module.

three-point function takes the form

$$
\left\langle\mathcal{O}_{\Delta_{1}}(\infty) \mathcal{O}_{\Delta_{2}}(\mathbf{u}) \mathcal{O}_{\Delta}(\mathbf{y})\right\rangle=\lim _{z \rightarrow \infty}\left(z^{2}\right)^{\Delta_{1}}\left\langle\mathcal{O}_{\Delta_{1}}(\mathbf{z}) \mathcal{O}_{\Delta_{2}}(\mathbf{u}) \mathcal{O}_{\Delta}(\mathbf{y})\right\rangle=\frac{1}{\left[(\mathbf{u}-\mathbf{y})^{2}\right]^{\frac{\Delta_{2}+\Delta-\Delta_{1}}{2}}}
$$

which can be expanded in terms of hyperspherical harmonics using (2.33) as

$$
\begin{aligned}
& \left\langle\mathcal{O}_{\Delta_{1}}(\infty) \mathcal{O}_{\Delta_{2}}(\mathbf{u}) \mathcal{O}_{\Delta}(\mathbf{y})\right\rangle \\
& \quad=\left(4 \pi^{d / 2}\right) \sum_{l=0}^{\infty} \sum_{s=0}^{\infty} \frac{\left(\frac{\Delta_{2}+\Delta-\Delta_{1}}{2}\right)_{l+s}\left(\frac{\Delta_{2}+\Delta-\Delta_{1}}{2}-\frac{d-2}{2}\right)_{s}}{s ! \Gamma(l+s+d / 2)}\left(y^{2}\right)^{s} \sum_{\mathbf{m}} M_{\mathbf{m}}^{l}(\mathbf{u}) M_{\mathbf{m}}^{l *}(\mathbf{y})
\end{aligned}
$$

Amputation of the out-going leg (2.46) ending at $\mathbf{y}$ from the above expression gives

$$
\left[\frac{4 \pi^{d / 2}}{s !(d / 2)_{l+s}(\Delta)_{l+s}\left(\Delta-\frac{d-2}{2}\right)_{s}}\right]^{\frac{1}{2}}\left(\frac{\Delta-\Delta_{12}}{2}\right)_{l+s}\left(\frac{\Delta-\Delta_{12}}{2}-\frac{d-2}{2}\right)_{s} M_{\mathbf{m}}^{l}(\mathbf{u})
$$

where $\Delta_{i j} \equiv \Delta_{i}-\Delta_{j}$. Similarly to find the other OPE module we start with the three-point function

$$
\left\langle\mathcal{O}_{\Delta}(\mathbf{y}) \mathcal{O}_{\Delta_{3}}(\mathbf{x}) \mathcal{O}_{\Delta_{4}}(\mathbf{0})\right\rangle=\left(y^{2}\right)^{\frac{\Delta_{3}-\Delta_{4}-\Delta}{2}}\left(x^{2}\right)^{\frac{\Delta-\Delta_{3}-\Delta_{4}}{2}} \frac{1}{\left[(\mathbf{y}-\mathbf{x})^{2}\right]^{\frac{\Delta+\Delta_{3}-\Delta_{4}}{2}}}
$$

Expanding this in hyperspherical harmonics gives

$$
\begin{aligned}
& \left\langle\mathcal{O}_{\Delta}(\mathbf{y}) \mathcal{O}_{\Delta_{3}}(\mathbf{x}) \mathcal{O}_{\Delta_{4}}(\mathbf{0})\right\rangle \\
& =\left(4 \pi^{d / 2}\right)\left(x^{2}\right)^{\frac{\Delta-\Delta_{3}-\Delta_{4}}{2}} \sum_{l, s=0}^{\infty} \frac{\left(\frac{\Delta+\Delta_{3}-\Delta_{4}}{2}\right)_{l+s}\left(\frac{\Delta+\Delta_{3}-\Delta_{4}}{2}-\frac{d-2}{2}\right)_{s}}{s ! \Gamma(l+s+d / 2)}\left(x^{2}\right)^{s}\left(y^{2}\right)^{\Delta-l-s} \\
& \quad \times \sum_{\mathbf{m}} M_{\mathbf{m}}^{l}(\mathbf{y}) M_{\mathbf{m}}^{l *}(\mathbf{x})
\end{aligned}
$$

Now amputating the in-going leg (2.44) starting from $\mathbf{y}$, we obtain

$$
\begin{aligned}
& \left(x^{2}\right)^{\frac{\left(\Delta-\Delta_{3}-\Delta_{4}\right)}{2}}\left[\frac{1}{4 \pi^{d / 2} s !(d / 2)_{l+s}(\Delta)_{l+s}\left(\Delta-\frac{d-2}{2}\right)_{s}}\right]^{\frac{1}{2}} \\
& \quad \times \frac{1}{\Gamma(d / 2)}\left(\frac{\Delta+\Delta_{34}}{2}\right)_{l+s}\left(\frac{\Delta+\Delta_{34}}{2}-\frac{d-2}{2}\right)_{s}\left(x^{2}\right)^{s} M_{\mathbf{m}}^{l *}(\mathbf{x})
\end{aligned}
$$




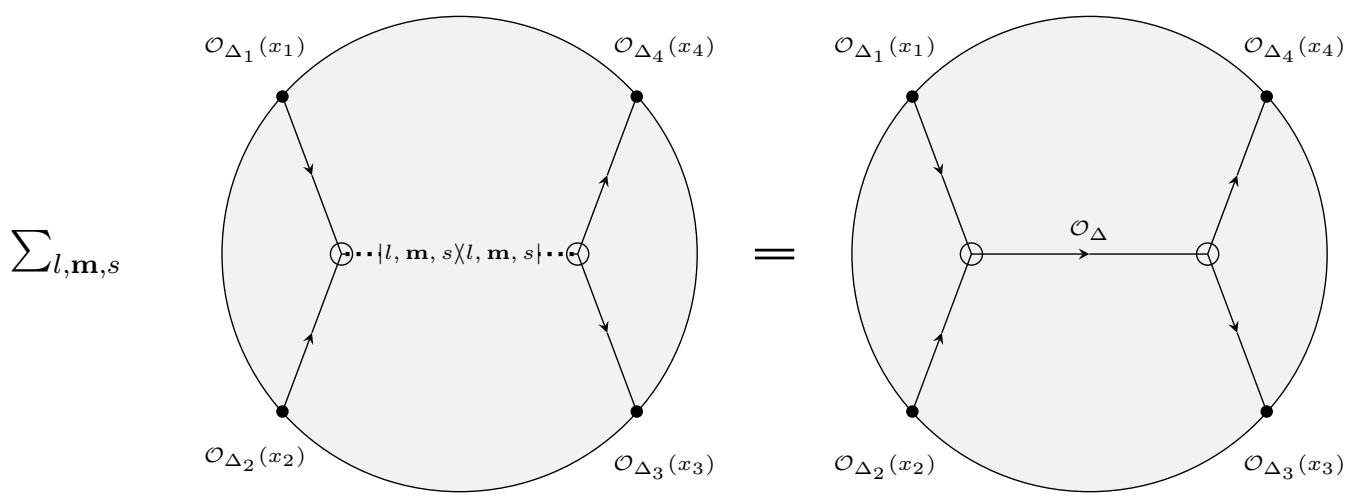

Figure 7. 4-point block from OPE modules.

Finally we glue the OPE modules (2.53) and (2.56) to compute the four-point conformal partial wave (see figure 7). Thus our prescription for the corresponding four-point conformal partial wave gives

$$
\begin{aligned}
W_{\Delta, 0}^{(d)}\left(\Delta_{i}, \mathbf{x}\right)= & \left(x^{2}\right)^{\frac{\left(\Delta-\Delta_{3}-\Delta_{4}\right)}{2}} \frac{1}{\Gamma(d / 2)} \sum_{l, s} \frac{\left(\frac{\Delta-\Delta_{12}}{2}\right)_{l+s}\left(\frac{\Delta+\Delta_{34}}{2}\right)_{l+s}}{s !(d / 2)_{l+s}(\Delta)_{l+s}\left(\Delta-\frac{d-2}{2}\right)_{s}} \\
& \times\left(\frac{\Delta-\Delta_{12}}{2}-\frac{d-2}{2}\right)_{s}\left(\frac{\Delta+\Delta_{34}}{2}-\frac{d-2}{2}\right)_{s}\left(x^{2}\right)^{s} \sum_{\mathbf{m}} M_{\mathbf{m}}^{l}{ }^{\star}(\mathbf{x}) M_{\mathbf{m}}^{l}(\mathbf{u})
\end{aligned}
$$

Using (2.27) we express this in terms of Gegenbauer polynomials

$$
\begin{aligned}
W_{\Delta, 0}^{(d)}\left(\Delta_{i}, \mathbf{x}\right)= & \left(x^{2}\right)^{\frac{\left(\Delta-\Delta_{3}-\Delta_{4}\right)}{2}} \frac{\Gamma\left(\frac{d-2}{2}\right)}{4 \pi^{d / 2} \Gamma(d / 2)} \sum_{l, s} \frac{(2 l+d-2)\left(\frac{\Delta-\Delta_{12}}{2}\right)_{l+s}\left(\frac{\Delta+\Delta_{34}}{2}\right)_{l+s}}{s !(d / 2)_{l+s}(\Delta)_{l+s}\left(\Delta-\frac{d-2}{2}\right)_{s}} \\
& \times\left(\frac{\Delta-\Delta_{12}}{2}-\frac{d-2}{2}\right)_{s}\left(\frac{\Delta+\Delta_{34}}{2}-\frac{d-2}{2}\right)_{s} x^{l+2 s} C_{l}^{\frac{d-2}{2}}\left(\frac{\mathbf{x} \cdot \mathbf{u}}{x}\right)
\end{aligned}
$$

This is our final result for the scalar conformal partial wave. Even though we assumed $d \geq 2$, we will see in the next section this result also holds for $d=1$. Notice that as advertised in the introduction our answer is naturally given in terms of Gegenbauer polynomials.

A result for the same quantity already exists in the literature in terms of the cross ratios [1]. In appendix B we show our answer agrees with their result.

In principle one can put together the conformal wave functions of section 2 , and the CGC of appendix A suitably to generate the scalar CPWs of any higher-point scalar correlators as well (as was done for $d=2$ case in [19]).

\section{Recovery of results in $d \leq 4$}

In this section we want to recover the known results for four-point scalar conformal partial waves in $d=1, \cdots, 4$ from our answer (2.58). For this we find it convenient to express our answer in different variables. Writing $\mathbf{x} \cdot \mathbf{u}=x \cos \theta$, we define

$$
z=x e^{i \theta}, \quad \bar{z}=x e^{-i \theta} .
$$


In terms of these variables $(z, \bar{z})$ the four-point CPW (2.58) takes the form

$$
\begin{aligned}
& W_{\Delta, 0}^{(d)}\left(\Delta_{i} ; z, \bar{z}\right)=(z \bar{z})^{\frac{\left(\Delta-\Delta_{3}-\Delta_{4}\right)}{2}} \frac{1}{4 \pi^{d / 2}} \sum_{l, s} \frac{(2 l+d-2)\left(\frac{\Delta-\Delta_{12}}{2}\right)_{l+s}\left(\frac{\Delta+\Delta_{34}}{2}\right)_{l+s}}{s !(d / 2)_{l+s}(\Delta)_{l+s}\left(\Delta-\frac{d-2}{2}\right)_{s}} \\
& \quad \times\left(\frac{\Delta-\Delta_{12}}{2}-\frac{d-2}{2}\right)_{s}\left(\frac{\Delta+\Delta_{34}}{2}-\frac{d-2}{2}\right)_{s}(z \bar{z})^{s+\frac{l}{2}} \frac{2}{(d-2)} C_{l}^{\frac{d-2}{2}}\left(\frac{z+\bar{z}}{2 \sqrt{z \bar{z}}}\right)
\end{aligned}
$$

$\boldsymbol{d}=4$. Substituting $d=4$ in (3.2) and manipulating further we find

$$
\begin{aligned}
& W_{\Delta, 0}^{(4)}\left(\Delta_{i}, z, \bar{z}\right)= \frac{1}{z-\bar{z}}(z \bar{z})^{\frac{1}{2}\left(\Delta-\Delta_{3}-\Delta_{4}\right)} \sum_{l, s=0}^{\infty} \Gamma\left(\frac{1}{2}\left(\Delta+\Delta_{34}\right)+l+s\right) \Gamma\left(\frac{1}{2}\left(\Delta-\Delta_{12}\right)+l+s\right) \\
& \times \Gamma\left(\frac{1}{2}\left(\Delta-\Delta_{12}\right)+s-1\right) \Gamma\left(\frac{1}{2}\left(\Delta+\Delta_{34}\right)+s-1\right) \\
& \times \frac{(l+1) \Gamma(\Delta) \Gamma(\Delta-1)}{s !(l+s+1) ! \Gamma(\Delta+l+s) \Gamma(\Delta+s-1)}\left(z^{l+s+1} \bar{z}^{s}-z^{s} \bar{z}^{l+s+1}\right) \\
&= \Gamma(\alpha) \Gamma(\alpha-1) \Gamma(\beta) \Gamma(\beta-1) \frac{1}{z-\bar{z}}(z \bar{z})^{\frac{1}{2}\left(\Delta-\Delta_{3}-\Delta_{4}\right)} \\
& \times\left[z{ }_{2} F_{1}(\alpha, \beta, \Delta, z){ }_{2} F_{1}(\alpha-1, \beta-1, \Delta-2, \bar{z})-\bar{z}{ }_{2} F_{1}(\alpha, \beta, \Delta, \bar{z}){ }_{2} F_{1}(\alpha-1, \beta-1, \Delta-2, z)\right]
\end{aligned}
$$

where $\alpha=\frac{1}{2}\left(\Delta-\Delta_{12}\right)$ and $\beta=\frac{1}{2}\left(\Delta+\Delta_{34}\right)$. The details of the calculation of how to go from the first to the second expression are relegated to appendix C. Our answer perfectly matches with the known results [1].

$\boldsymbol{d}=3$. When $d=3$ the Gegenbauer polynomials used to express the answer (3.2) become the Legendre polynomials, i.e. $C_{l}^{1 / 2}(\cos \theta)=P_{l}(\cos \theta)$. Therefore, our answer reads

$$
\begin{aligned}
W_{\Delta, 0}^{(3)}\left(\Delta_{i} ; z, \bar{z}\right)= & (z \bar{z})^{\frac{\left(\Delta-\Delta_{3}-\Delta_{4}\right)}{2}} \frac{1}{\pi^{3 / 2}} \sum_{l, s=0}^{\infty} \frac{(l+1 / 2)\left(\frac{\Delta-\Delta_{12}}{2}\right)_{l+s}\left(\frac{\Delta+\Delta_{34}}{2}\right)_{l+s}}{s !(3 / 2)_{l+s}(\Delta)_{l+s}(\Delta-1 / 2)_{s}} \\
& \times\left(\frac{\Delta-\Delta_{12}}{2}-\frac{1}{2}\right)_{s}\left(\frac{\Delta+\Delta_{34}}{2}-\frac{1}{2}\right)_{s}(z \bar{z})^{s+\frac{l}{2}} P_{l}\left(\frac{z+\bar{z}}{2 \sqrt{z \bar{z}}}\right)
\end{aligned}
$$

We are not aware of any closed form for this case. There exists a conjectured formula by [39] where the $d=3$ four-point block is written in terms of $2 d$ blocks. We have checked that our answer also agrees with [39] for large ranges of $l$ and $s$.

$\boldsymbol{d}=\mathbf{2}$. To recover the answer for $d=2$ we have to take the $d \rightarrow 2$ limit of (3.2). We find

$$
\begin{aligned}
W_{\Delta, 0}^{(2)}\left(\Delta_{i} ; z, \bar{z}\right)= & (z \bar{z})^{\frac{\left(\Delta-\Delta_{3}-\Delta_{4}\right)}{2}} \frac{1}{\pi} \sum_{l, s=0}^{\infty} \frac{\left(\frac{\Delta-\Delta_{12}}{2}\right)_{l+s}\left(\frac{\Delta+\Delta_{34}}{2}\right)_{l+s}}{s !(l+s) !(\Delta)_{l+s}(\Delta)_{s}}\left(\frac{\Delta-\Delta_{12}}{2}\right)_{s}\left(\frac{\Delta+\Delta_{34}}{2}\right)_{s} \\
& \times(z \bar{z})^{s+\frac{l}{2}} \cos (l \theta) \\
= & \frac{1}{2 \pi}(z \bar{z})^{\frac{\left(\Delta-\Delta_{3}-\Delta_{4}\right)}{2}} \sum_{l, s=0}^{\infty} \frac{\left(\frac{\Delta-\Delta_{12}}{2}\right)_{l+s}\left(\frac{\Delta+\Delta_{34}}{2}\right)_{l+s} \frac{\left(\frac{\Delta-\Delta_{12}}{2}\right)_{s}\left(\frac{\Delta+\Delta_{34}}{2}\right)_{s}}{s !(\Delta)_{s}}}{(l+s) !(\Delta)_{l+s}} \frac{\times\left(z^{l+s} \bar{z}^{s}+z^{s} \bar{z}^{l+s}\right)}{}
\end{aligned}
$$


where we have used the following identity

$$
\lim _{\mu \rightarrow 0} \frac{1}{\mu} C_{l}^{\mu}(\cos \theta)=\frac{2}{l} T_{l}(\cos \theta)=\frac{2}{l} \cos (l \theta)
$$

where $T_{l}$ are Chebyshev polynomials of the first kind. Finally performing the summations we recover the familiar answer for scalar CPW in two dimensions

$$
\begin{aligned}
W_{\Delta, 0}^{(2)}\left(\Delta_{i} ; z, \bar{z}\right)= & \frac{1}{\pi}(z \bar{z})^{\frac{1}{2}\left(\Delta-\Delta_{3}-\Delta_{4}\right)}{ }_{2} F_{1}\left[\left(\frac{\Delta-\Delta_{12}}{2}\right),\left(\frac{\Delta+\Delta_{34}}{2}\right) ; \Delta ; z\right] \\
& \times{ }_{2} F_{1}\left[\left(\frac{\Delta-\Delta_{12}}{2}\right),\left(\frac{\Delta+\Delta_{34}}{2}\right) ; \Delta ; \bar{z}\right]
\end{aligned}
$$

$\boldsymbol{d}=\mathbf{1}$. This case corresponds to $\frac{d-2}{2}=-1 / 2$ and the corresponding Gegenbauer polynomials take the following form:

$$
C_{l}^{-\frac{1}{2}}(\chi)=\delta_{l, 0}-\chi \delta_{l, 1}+\theta(l-2) \frac{1-\chi^{2}}{l(l-1)} \frac{d}{d \chi} P_{l-1}(\chi)
$$

Further, in this case all the positions of the operators are simply real numbers. In particular, the unit vector $\mathbf{u}$ becomes either 1 or -1 . Without loss of generality we take $\mathbf{u}=1$. Then the argument of the Gegenbauer polynomials in (2.58), $\hat{\mathbf{x}} \cdot \mathbf{u}$ also becomes \pm 1 depending on the sign of $\mathbf{x}$. For both the cases the Gegenbauer polynomial simplifies to

$$
C_{l}^{(-1 / 2)}( \pm 1)=\delta_{l 0} \mp \delta_{l 1}=\delta_{l 0}-\operatorname{sign}(\mathbf{x}) \delta_{l 1}
$$

Then the expression for 4-point CPW splits into two parts as follows

$$
\begin{aligned}
W_{\Delta, 0}^{(1)}\left(\Delta_{i}, x\right)= & \left(x^{2}\right)^{\frac{\left(\Delta-\Delta_{3}-\Delta_{4}\right)}{2}} \frac{1}{2 \sqrt{\pi}}\left[\sum_{s=0}^{\infty} \frac{(\alpha)_{s}(\beta)_{s}}{s !(1 / 2)_{s}(\Delta)_{s}\left(\Delta+\frac{1}{2}\right)_{s}}\left(\alpha+\frac{1}{2}\right)_{s}\left(\beta+\frac{1}{2}\right)_{s} x^{2 s}\right. \\
& \left.+\operatorname{sign}(x) \sum_{s=0}^{\infty} \frac{(\alpha)_{s+1}(\beta)_{s+1}}{s !(1 / 2)_{s+1}(\Delta)_{s+1}\left(\Delta+\frac{1}{2}\right)_{s}}\left(\alpha+\frac{1}{2}\right)_{s}\left(\beta+\frac{1}{2}\right)_{s} x^{2 s+1}\right]
\end{aligned}
$$

where $\alpha=\frac{1}{2}\left(\Delta-\Delta_{12}\right)$ and $\beta=\frac{1}{2}\left(\Delta+\Delta_{34}\right)$ as before. Now using the following identities for Pochhammer symbols

$$
(A)_{s}\left(A+\frac{1}{2}\right)_{s}=\frac{1}{2^{2 s}}(2 A)_{2 s}, \quad(A)_{s+1}\left(A+\frac{1}{2}\right)_{s}=\frac{1}{2^{2 s+1}}(2 A)_{2 s+1}
$$

for $A \in\{\alpha, \beta, \Delta\}$, and

$$
s !\left(\frac{1}{2}\right)_{s}=\frac{(2 s) !}{2^{2 s}}, \quad s !\left(\frac{1}{2}\right)_{s+1}=\frac{(2 s+1) !}{2^{2 s+1}}
$$

we can show that the expression (3.9) can be written as a single sum, which can be carried out to yield the answer

$$
W_{\Delta, 0}^{(1)}(x)=\frac{1}{2 \sqrt{\pi}} x^{\Delta-\Delta_{3}-\Delta_{4}}{ }_{2} F_{1}(2 \alpha, 2 \beta ; 2 \Delta ; x)
$$

where $x=|\mathbf{x}|$. This expression agrees with the known result $[40,41]$ for the $d=1$ case. 


\section{Seed blocks and recursion relations}

There exist in the literature some powerful recursion relations that enable one to compute the CPW in a given dimension in terms of those in lower dimensions [4, 38, 39]. For instance, one such recursion relation among the even dimensional CPWs was given in [4]. In this section we give a different (and simpler) proof of this relation using our answer, and provide a counterpart of such a relation among the odd dimensional CPWs. For this we begin by extracting the conformal block from the CPW via the relation: $W_{\Delta, 0}^{(d)}\left(\Delta_{i}, \mathbf{x}\right):=$ $\left(x^{2}\right)^{-\frac{1}{2}\left(\Delta_{3}+\Delta_{4}\right)} G_{\Delta}^{\mu}(\alpha, \beta ; \mathbf{x})$ where $\alpha=\frac{1}{2}\left(\Delta-\Delta_{12}\right), \beta=\frac{1}{2}\left(\Delta+\Delta_{34}\right)$ and $\mu=\frac{d-2}{2}$. Then from (2.58) we have:

$$
G_{\Delta}^{\mu}(\alpha, \beta ; \mathbf{x})=\left(x^{2}\right)^{\frac{\Delta}{2}} \sum_{l, s=0}^{\infty} \frac{(\alpha)_{l+s}(\beta)_{l+s}(\alpha-\mu)_{s}(\beta-\mu)_{s}}{s !(\Delta)_{l+s}(\mu+1)_{l+s}}\left(1+\frac{l}{\mu}\right) x^{l+2 s} C_{l}^{\mu}(\cos \theta) .
$$

Differentiating with respect to $\cos \theta$ and using the identity

$$
\frac{d}{d z} C_{l}^{\mu}(z)=2 \mu C_{l-1}^{\mu+1}(z)
$$

(4.1) becomes

$$
\begin{aligned}
& \frac{d G_{\Delta}^{\mu}(\alpha, \beta ; \mathbf{x})}{d(\cos \theta)}=\left(x^{2}\right)^{\frac{\Delta}{2}} \sum_{l=1}^{\infty} \sum_{s=0}^{\infty} \frac{(\alpha)_{l+s}(\beta)_{l+s}(\alpha-\mu)_{s}(\beta-\mu)_{s}}{s !(\Delta)_{l+s}(\mu+1)_{l+s}}\left(1+\frac{l}{\mu}\right) x^{l+2 s} 2 \mu C_{l-1}^{\mu+1}(\cos \theta) \\
& =\frac{2 \alpha \beta}{\Delta}\left(x^{2}\right)^{\frac{\Delta+1}{2}} \sum_{l, s=0}^{\infty} \frac{(\alpha+1)_{l+s}(\beta+1)_{l+s}(\alpha-\mu)_{s}(\beta-\mu)_{s}}{s !(\Delta+1)_{l+s}(\mu+2)_{l+s}}\left(1+\frac{l}{\mu+1}\right) x^{l+2 s} C_{l}^{\mu+1}(\cos \theta) \\
& =\frac{2 \alpha \beta}{\Delta} G_{\Delta+1}^{\mu+1}(\alpha+1, \beta+1 ; \mathbf{x})
\end{aligned}
$$

where, in going from the first to the second line we have replaced $l \rightarrow l+1$ and used the identity: $(\alpha)_{n+1}=\alpha(\alpha+1)_{n}$. By applying this relation repeatedly (say $k$ times) we arrive at:

$$
\begin{aligned}
G_{\Delta}^{\mu}(\alpha, \beta ; \mathbf{x}) & =\frac{(\Delta-k)_{k}}{2^{k}(\alpha-k)_{k}(\beta-k)_{k}}\left(\frac{d}{d \cos \theta}\right)^{k} G_{\Delta-k}^{\mu-k}(\alpha-k, \beta-k ; \mathbf{x}) \\
& =\frac{(\alpha)_{-k}(\beta)_{-k}}{2^{k}(\Delta)_{-k}}\left(\frac{d}{d \cos \theta}\right)^{k} G_{\Delta-k}^{\mu-k}(\alpha-k, \beta-k ; \mathbf{x})
\end{aligned}
$$

where we have used $(\alpha-k)_{k}(\alpha)_{-k}=1$. Since $\mu \rightarrow \mu+1$ corresponds to $d \rightarrow d+2$ the equation (4.4) says that we can get all even (odd) dimensional conformal blocks starting from, say the $2 d(3 d)$ blocks. Writing $d=2 k+2+2 \gamma$ where $\gamma=0$ for even $d$ and $\gamma=1 / 2$ for odd $d$, we can recast this result as

$$
G_{\Delta}^{k+\gamma}(\alpha, \beta ; \mathbf{x})=\frac{(\alpha)_{-k}(\beta)_{-k}}{(\Delta)_{-k}}\left(\frac{d}{d v}\right)^{k} G_{\Delta-k}^{\gamma}(\alpha-k, \beta-k ; \mathbf{x})
$$

where using (3.1) we have defined

$$
\frac{d}{d v}=\frac{x}{z-\bar{z}}\left(z \frac{d}{d z}-\bar{z} \frac{d}{d \bar{z}}\right) .
$$

This result for the case of $\gamma=0$ (relating different even dimensional blocks) is the one found in [4] — whereas the case of $\gamma=1 / 2$ is its odd dimensional counterpart. 


\section{Some odds and ends}

In this section we present a couple of additional results that are a selection of possible generalisations in various directions of the cases considered so far. One of the limitations is the restriction to scalar operators (both in the external and the internal legs). The cases of $d=1$ and $d=2$ are the simplest to address in this regard. The $d=2$ case was solved completely in [19]. The $d=1$ case can also be treated in full generality, which we present here.

\subsection{Complete $d=1$ analysis}

First we would like to compute the cap state for $1 d$ case and then the $1 d$ global blocks. We begin with the infinite dimensional matrix representations [42] of global conformal algebra $\operatorname{sl}(2, \mathbf{R})$ for $\mathrm{CFT}_{1}$ :

$$
\begin{aligned}
& L_{1}|h, n\rangle=\sqrt{n(2 h+n-1)}|h, n-1\rangle, \quad L_{-1}|h, n\rangle=\sqrt{(n+1)(2 h+n)}|h, n+1\rangle, \\
& L_{0}|h, n\rangle=(h+n)|h, n\rangle
\end{aligned}
$$

where $D=L_{0}, P=L_{-1}$ and $K=L_{1}$. The bulk is the $\mathbb{H}^{2}$ space whose tangent space rotation group is $\mathrm{SO}(2)$. Therefore, the cap state $|h, \theta\rangle\rangle$ transforms as a 1-dimensional irrep of $\mathrm{SO}(2)$ :

$$
\left.\left.\left(L_{1}-L_{-1}\right)|h, \theta\rangle\right\rangle=\theta|h, \theta\rangle\right\rangle
$$

The parameter $\theta$, a purely imaginary number, is related to the spin of the general bulk field - we will elaborate further on this shortly. This equation can be solved for $|h, \theta\rangle\rangle$ as a linear combination of states in the module:

$$
|h, \theta\rangle\rangle=\sum_{n=0}^{\infty} C_{n}|h, n\rangle,
$$

writing $C_{n}=\sqrt{\frac{\Gamma(2 h)}{n ! \Gamma(2 h+n)}} f_{n}$ with the $f_{n}$ satisfying the recursion relation

$$
f_{n+1}=\theta f_{n}+n(2 h+n-1) f_{n-1} .
$$

It is not difficult to see that the $f_{n}$ are generated by $G(x)=\sum_{n=0}^{\infty} \frac{x^{n}}{n !} f_{n}$ where

$$
G(x)=(1-x)^{-h-\frac{\theta}{2}}(1+x)^{-h+\frac{\theta}{2}} .
$$

We find the coefficient of $\frac{x^{n}}{n !}$ in $G(x)$ to be:

$$
f_{n}=(-1)^{n}\left(h-\frac{\theta}{2}\right)_{n}{ }_{2} F_{1}\left(-n, h+\frac{\theta}{2},-h+\frac{\theta}{2}-n+1 ;-1\right) .
$$

Having obtained the expression for the most general cap state in $d=1$, we can repeat the rest of the exercises carried out in section 2 on these caps. Working with the coset element $g(x)=e^{\rho L_{0}} e^{-x L_{-1}}$ we can extract the leading terms in the large- $\rho$ limit of $\langle\langle h, \theta|g(x)| h, k\rangle$ and $\left.\left\langle h, k\left|g^{-1}(y)\right| h, \theta\right\rangle\right\rangle$. With some further analysis we find the following simple answers in the $\rho \rightarrow \infty$ limit:

$$
\lim _{\rho \rightarrow \infty} e^{\rho h}\left\langle\langle h, \theta|g(x)| h, k\rangle=(-1)^{-h-\frac{\theta}{2}} \sqrt{\frac{\Gamma(2 h+k)}{k ! \Gamma(2 h)}} x^{-2 h-k}\right.
$$




$$
\left.\lim _{\rho \rightarrow \infty} e^{\rho h}\left\langle h, k\left|g^{-1}(y)\right| h, \theta\right\rangle\right\rangle=y^{k} \sqrt{\frac{\Gamma(2 h+k)}{k ! \Gamma(2 h)}}
$$

Notice that even though the general cap states depend on the spin-parameter $\theta$ the final expressions (5.7) for the legs have essentially no dependence on it. For example, putting the legs together and performing the sum over $k$ gives

$$
\lim _{\rho \rightarrow \infty} e^{2 \rho h}\left\langle\left\langle h, \theta\left|g(x) g^{-1}(y)\right| h, \theta\right\rangle\right\rangle=(-1)^{-h-\frac{\theta}{2}} x^{-2 h} \sum_{k=0}^{\infty} \frac{\Gamma(2 h+k)}{k ! \Gamma(2 h)}\left(\frac{x}{y}\right)^{k}=(-1)^{-h-\frac{\theta}{2}} \frac{1}{(x-y)^{2 h}}
$$

A comparison of the $d=1$ legs here with the holomorphic part of the $d=2$ case of [19] enables us to immediately write down the $1 d$ blocks by starting with the holomorphic parts of $d=2$ blocks and replacing $h \rightarrow \Delta$ and $z \rightarrow|x|$. It is evident that this will give rise to the 4-point block found above (3.12), and higher-point ones to match with [40, 41].

The interpretation of $\boldsymbol{\theta}$. To better understand the role of $\theta$ we must first look at the linearised bulk equations satisfied by the legs $\left.\left\langle h, k\left|g^{-1}(x)\right| h, \theta\right\rangle\right\rangle$ and $\langle\langle h, \theta|g(x)| h, k\rangle$. To this end we first list the following identities [19] satisfied by $g^{-1}(x)$

$$
\begin{aligned}
& L_{0} g^{-1}(x)=\left(-\partial_{\rho}+x \partial_{x}\right) g^{-1}(x), L_{-1} g^{-1}(x)=-\partial_{x} g^{-1}(x), \\
& L_{1} g^{-1}(x)=\left(2 x \partial_{\rho}-x^{2} \partial_{x}+e^{-\rho} \partial_{x}\right) g^{-1}(x)+g^{-1}(x) e^{-\rho}\left(L_{1}-L_{-1}\right) .
\end{aligned}
$$

Using these we can easily compute the action of the $\operatorname{sl}(2, \mathbf{R})$ Casimir operator $C_{2}$ on $g^{-1}(x)$

$$
\begin{aligned}
C_{2} & =2 L_{0}^{2}-L_{1} L_{-1}-L_{-1} L_{1} \\
C_{2} g^{-1}(x) & =2\left(\partial_{\rho}^{2}+\partial_{\rho}+e^{-2 \rho} \partial_{x}^{2}\right) g^{-1}(x)+e^{-\rho} \partial_{x} g^{-1}(x)\left(L_{1}-L_{-1}\right)
\end{aligned}
$$

Thus we see that the legs $\left.\left\langle h, k\left|g^{-1}(x)\right| h, \theta\right\rangle\right\rangle$ satisfy the second order PDE:

$$
\left.\left.\left(\partial_{\rho}^{2}+\partial_{\rho}+e^{-2 \rho} \partial_{x}^{2}+\theta e^{-\rho} \partial_{x}\right)\left\langle h, k\left|g^{-1}(x)\right| h, \theta\right\rangle\right\rangle=\Delta(\Delta-1)\left\langle h, k\left|g^{-1}(x)\right| h, \theta\right\rangle\right\rangle .
$$

It is not difficult to see that the other legs $\langle\langle h, \theta|g(x)| h, k\rangle$ also satisfy the same equation. We would now like to interpret this equation as that of a bulk local field in the background $A d S_{2}$ geometry with metric $d s_{\mathbb{H}^{2}}^{2}=d \rho^{2}+e^{2 \rho} d x^{2}$.

Since the boundary isometry group is just $\mathbb{Z}_{2}$ we would expect the boundary conformal primary operators to be characterised by a scaling dimension $\Delta$ and a parity \pm 1 . But any general bulk local field in two dimensions (once one trades off the spacetime indices for the tangent space ones) has to have only two parameters: the mass and the spin on which the bulk covariant derivative acts as

$$
D_{\mu} \psi(x)=\partial_{\mu} \psi(x)+\frac{1}{2} \omega_{\mu}^{a b} L_{a b} \psi(x)
$$

where $L_{a b}$ is the tangent space rotation generator in the representation of $\psi(x)$. Redefining the coordinates $z=e^{-\rho}+i x, \bar{z}=e^{-\rho}-i x$ the metric of $A d S_{2}$ becomes $d s^{2}=\frac{4 d z d \bar{z}}{(z+\bar{z})^{2}}$. For this geometry we have the following non-zero vielbeins, spin-connections and Christoffel connections:

$$
e^{+}=\frac{2 d z}{(z+\bar{z})^{2}}, \quad e^{-}=\frac{2 d \bar{z}}{(z+\bar{z})^{2}}, \quad \omega^{+}+\frac{d z-d \bar{z}}{(z+\bar{z})}=-\omega_{-}^{-}, \quad \Gamma_{z z}^{z}=\frac{-2}{z+\bar{z}}=\Gamma_{\bar{z} \bar{z}}^{\bar{z}}
$$


Since the tangent space is just $\mathbb{R}^{2}$, there is only one rotation generator $L_{+-}$, and we can take the field $\psi(x)$ to be an eigenstate of it with eigenvalue $i \theta$. Then it is easy to show that such a field satisfies the following

$$
\left(\square-m^{2}\right) \psi(x)=\left(\partial_{\rho}^{2}+\partial_{\rho}+e^{-2 \rho} \partial_{x}^{2}+\theta e^{-\rho} \partial_{x}\right) \psi(x)-\left(m^{2}+\frac{1}{8} \theta^{2}\right) \psi(x)=0
$$

Comparing (5.11) and (5.14) we make the following identifications:

$$
\Delta(\Delta-1)=m^{2}+\frac{\theta^{2}}{8} .
$$

Therefore, we conclude that, when it is available, the parameter $\theta$ represents the spin of the bulk field. ${ }^{8}$

\section{$5.2 \mathrm{OWN} s$ in more general $\boldsymbol{A d S}_{3}$ geometries}

In [19] we provided the computation of $\mathrm{CPW}$ of vacuum correlators in $\mathrm{CFT}_{2}$ of primaries in general representations of the conformal algebra using the Wilson network prescription. Here we extend this result to include CPW of correlators in any (heavy) state, and to thermal correlators. This involves computing the OWN in appropriate locally $A d S_{3}$ geometries. Recall that in Fefferman-Graham gauge the most general solution to $A d S_{3}$ gravity [43] is

$$
\begin{aligned}
l^{-2} d s^{2}= & d \rho^{2}+\left(d x_{1}^{2}+d x_{2}^{2}\right)\left(e^{2 \rho}+T(z) \bar{T}(\bar{z}) e^{-2 \rho}\right) \\
& +(T(z)+\bar{T}(\bar{z}))\left(d x_{1}^{2}-d x_{2}^{2}\right)+2 i(T(z)-\bar{T}(\bar{z})) d x_{1} d x_{2}
\end{aligned}
$$

When $-\infty<x_{i}<\infty-$ it is a Euclidean locally $A d S_{3}$ geometry with boundary $\mathbb{R}^{2}$. For constant values of $T, \bar{T} \geq 0$ these are interpreted as BTZ black holes. When $-1 / 4<T, \bar{T}<$ 0 these represent heavy CFT states. We restrict to the constant $T, \bar{T}$ cases from now on. The relevant coset element is

$$
g(x)=e^{\rho\left(L_{0}+\bar{L}_{0}\right)} e^{-z\left(L_{-1}-T L_{1}\right)} e^{-\bar{z}\left(\bar{L}_{-1}-\bar{T} \bar{L}_{1}\right)}
$$

One can carry out the rest of the computations following [19]. We find that the expressions for legs in the $\rho \rightarrow \infty$ are:

$$
\begin{aligned}
\lim _{\rho \rightarrow \infty} e^{\rho(h+\bar{h})}\langle\langle h, \bar{h} ; j, m|g(x)| h, \bar{h} ; k, \bar{k}\rangle= & \frac{\lambda(-1)^{h-\bar{h}}}{\Gamma(2 h) \sqrt{\Gamma(2 h-2 \bar{h})}}\left(\frac{\sqrt{T}}{\sinh (z \sqrt{T})}\right)^{2 h}\left(\frac{\sqrt{\bar{T}}}{\sinh (\bar{z} \sqrt{\bar{T}})}\right)^{2 \bar{h}} \\
& \times \sqrt{\frac{\Gamma(2 h+k)}{k !} \frac{\Gamma(2 \bar{h}+\bar{k})}{\bar{k} !}\left(\frac{\sqrt{T}}{\tanh (z \sqrt{T})}\right)^{k}\left(\frac{\sqrt{\bar{T}}}{\tanh (\bar{z} \sqrt{\bar{T}})}\right)^{\bar{k}}} \\
\left.\lim _{\rho \rightarrow \infty} e^{\rho(h+\bar{h})}\left\langle h, \bar{h} ; k, \bar{k}\left|g^{-1}(x)\right| h, \bar{h} ; j,-j\right\rangle\right\rangle= & \frac{\lambda}{\Gamma(2 h) \sqrt{\Gamma(2 j+1)}} \\
& \times \frac{\left(\frac{\tanh (z \sqrt{T})}{\sqrt{T}}\right)^{k}\left(\frac{\tanh (\bar{z} \sqrt{\bar{T}})}{\sqrt{\bar{T}}}\right)^{\bar{k}}}{(\cosh (z \sqrt{T}))^{2 h}(\cosh (\bar{z} \sqrt{\bar{T}}))^{2 \bar{h}}} \sqrt{\frac{\Gamma(2 h+k) \Gamma(2 \bar{h}+\bar{k})}{k ! \bar{k} !}}
\end{aligned}
$$

\footnotetext{
${ }^{8}$ Amusingly the same equation (5.14) arises for a complex scalar in $A d S_{2}$ minimally coupled to a background electric field preserving its isometries, and with strength $\theta$.
} 
Putting them together for the 2-point function yields:

$$
\left\langle\mathcal{O}_{(h, \bar{h})}\left(x_{1}\right) \mathcal{O}_{(h, \bar{h})}\left(x_{2}\right)\right\rangle_{(T, \bar{T})}=\left(\frac{\sqrt{T}}{\sinh \left(\left(z_{2}-z_{1}\right) \sqrt{T}\right)}\right)^{2 h}\left(\frac{\sqrt{\bar{T}}}{\sinh \left(\left(\bar{z}_{2}-\bar{z}_{1}\right) \sqrt{\bar{T}}\right)}\right)^{2 \bar{h}} .
$$

which is the well known two-point function of a thermal CFT [44, 45] (see also [46] and more recently [32]). The higher-point blocks can also be computed for these geometries [47].

\section{Discussion}

In this paper we have continued to develop further our prescription [19] to compute the conformal partial waves of CFT correlation functions using the gravitational open Wilson network operators in the holographic dual gravity theories. In particular, we have demonstrated how to use gravitational Open Wilson Networks to compute 4-point scalar partial waves (both external and the exchanged operators being scalars) in any dimension. As in [19] our prescription for scalar partial waves in general $d \geq 2$ also can be summarised into Feynman-like rules involving conformal wave functions (2.44), (2.46) and the ClebschGordan coefficients of the relevant conformal group.

Notice that we computed the legs first for generic bulk point and then took the boundary limit. Once we have obtained the results in (2.44), (2.46) the rest of the construction of the CPW does not have any further reference to the bulk. ${ }^{9}$ Indeed the Cap states we have used are the higher dimensional analogs of the Ishibashi states of the 2d CFTs, and as we had shown in [19] they can be used to construct the bulk local states - the CFT states corresponding to the HKLL type bulk local operators [55] (see also [17] for such states that go beyond the global conformal symmetry in $2 \mathrm{~d}$ ). One could leave some of the external points of the OWN in the bulk, and they should correspond to partial waves of correlators involving (boundary) local primaries as well as HKLL type bulk local operators. It will be interesting to compute such partial waves as well within our formalism.

Our result for the scalar CPW are naturally given in the Gegenbauer polynomial basis. We have compared our results with the known answers wherever available and found complete agreement. Our methods also lead to a simpler proof of the recursion relation of [4] in even dimensional CFTs, and lead to analogous recursion relations for odd dimensions.

The CPW for correlation functions of any primary with any exchanged operator has already been achieved in $d=2$ case in [19] and here in (in the simpler case of) $d=1$. It remains to generalise these computational techniques to obtain the CPWs for arbitrary representations in $d \geq 3 .{ }^{10}$ This involves finding the relevant cap states, and from there the relevant legs (conformal wave functions), and OPE modules etc. This work is in progress [48] and we hope to report on it in the near future. So far we have found the caps states for vectors, rank-2 antisymmetric and symmetric traceless tensor representations,

\footnotetext{
${ }^{9}$ It will be interesting to explore if it is possible to formulate the Wilson network computation such that the external points are anchored on the boundary from the beginning.

${ }^{10}$ The generalisation of geodesic Witten diagrams to include some of the other representations was achieved in $[10,11,16,18]$.
} 
and working on finding others. For those who may be interested, we present here the expressions of the cap states for the vector representation of the tangent space rotation algebra $s o(d+1)$. This is constructed as a linear combination of the basis elements of the conformal module over a vector primary state and it is given by:

$$
\begin{aligned}
\left.\left|\phi_{\Delta}, \alpha\right\rangle\right\rangle & =\sum_{n=0}^{\infty}\left(P_{\gamma} P^{\gamma}\right)^{n} \sum_{\beta=1}^{d} A_{\alpha \beta}^{(n)}(\Delta, d)\left|\phi_{\Delta}, \beta\right\rangle+\sum_{n=0}^{\infty}\left(P_{\gamma} P^{\gamma}\right)^{n} B^{(n)}(\Delta, d) P_{\alpha} \sum_{\gamma=1}^{d} P_{\gamma}\left|\phi_{\Delta}, \gamma\right\rangle \\
\left.\left|\phi_{\Delta}, d+1\right\rangle\right\rangle & =\sum_{n=0}^{\infty} C^{(n)}(\Delta, d)\left(P_{\gamma} P^{\gamma}\right)^{n} \sum_{\beta=1}^{d} P_{\beta}\left|\phi_{\Delta}, \beta\right\rangle
\end{aligned}
$$

with $A^{(n)}=C^{(n)}\left(\Delta_{\phi}-1\right)$ and $B^{(n)}=\frac{C^{(n)}}{2}+2(n+1) C^{(n+1)}\left(\Delta_{\phi}+n-\frac{d}{2}+2\right)$.

Of course one would like to see if our method gives answers in forms more amenable to potential applications, such as in the bootstrap approach towards the classification of CFTs. Since our answers are in Gegenbauer polynomial basis it is possible that they may be found more suitable - as working with this basis is much simpler (as we have seen in section 4 , for example).

An interesting set of future directions should include exploring the role of Weight shifting operators [49-51] in our formalism.

The CPW computed here are the analogs of the global conformal blocks of the $2 \mathrm{~d}$ $\mathrm{CFT} s$, and in the $2 \mathrm{~d}$ case the OWN $s$ have been generalised to compute the (finite- $c$ ) Virasoro blocks [21]. It will be interesting to ask if one can find quantum corrections to the classical OWN $s$ due to gravitational interaction/back reaction even in higher dimensions.

It may be of interest to compute objects similar to our OWNs in both flat and de Sitter gravity theories. Such diagrams could provide a basis of partial waves for S-matrices for scattering problems in these spaces.

Another possible generalisation should involve inclusion of boundaries and defects to the CFT [52-54] in the formalism considered.

We hope that this program will naturally lend itself to answering dynamical questions as well in CFTs.

\section{Acknowledgments}

We would like to thank ICTS, Bengaluru for hospitality during the final stages of this work, and the participants of the workshop "AdS/CFT @ 20 and Beyond" for discussions. AB would like to thank IoP, Bhubaneswar for hospitality while the work was in progress.

\section{A CGC required for scalar CPW}

Here we record results of CGC of the representations considered in the text for the algebra $s o(1, d+1)$. These are needed to compute the CPW for higher-point functions from OWNs. Before we present the detailed derivation of the CGC from the 3-point functions we collect a few facts about the irreducible representations of $s o(d)$ which we will use in the extraction of the Clebsch-Gordan coefficients. 
A finite dimensional irreducible representation of $s o(d)$ is uniquely defined by its highest weight $\left[\mu_{1}, \mu_{2}, \ldots \mu_{k}\right]$ with

$$
\begin{aligned}
& \mu_{1} \geq \mu_{2} \geq \cdots \geq \mu_{k-1} \geq\left|\mu_{k}\right| \quad \text { for } d=2 k \\
& \mu_{1} \geq \mu_{2} \geq \cdots \geq \mu_{k-1} \geq \mu_{k} \geq 0 \quad \text { for } d=2 k+1
\end{aligned}
$$

The components $\mu_{i}$ are either simultaneously integers (tensorial representations) or halfintegers (spinorial representations). We only consider symmetric traceless representations of $s o(d)$ as these are the only relevant ones for the scalar CGC of $s o(d+1,1)$. These could be represented on the Hilbert space $H$ of square integrable function on $S^{d-1}$. The Hilbert space can be decomposed into an orthogonal sum of subspaces $H^{l}$ of homogenous polynomials of degree $l$ in $d$ variables. We introduce a complete orthonormal basis $|l, \mathbf{M}\rangle$ on $H^{l}$, where $\mathbf{M}=\left(m_{d-2}, m_{d-3}, \ldots, m_{2}, m_{1}\right)$ label these basis states provided they fulfil:

$$
l=m_{d-1} \geq m_{d-2} \geq \cdots \geq m_{2} \geq\left|m_{1}\right| \quad m_{1} \in \mathbb{Z} \quad m_{i} \in \mathbb{Z}_{>0} \quad i \geq 2
$$

The dimension of the space $H$ is $d_{l}=(2 l+d-2) \frac{(l+d-3) !}{l !(d-2) !}$ - the number of independent components of a general symmetric traceless tensor of rank $l$ in $d$ dimensions. The matrix elements of the representation $D^{l}$ read:

$$
D_{\mathbf{M ~ M}^{\prime}}^{l}(g)=\left\langle l, \mathbf{M}\left|D^{l}(g)\right| l, \mathbf{M}^{\prime}\right\rangle
$$

In particular,

$$
D_{\mathbf{M} \mathbf{0}}^{l}(g)=\frac{1}{\sqrt{d_{l}}} N_{l \mathbf{M}}^{d} \prod_{k=1}^{d-2} C_{m_{k+1}-m_{k}}^{m_{k}+k / 2} \cos \left(\Phi_{k+1}\right) \sin ^{m_{k}}\left(\Phi_{k+1}\right) e^{i m_{1} \Phi_{1}}
$$

where $N_{l \mathrm{M}}^{d}$ is the normalisation w.r.t. the Haar measure on $s o(d), C_{\lambda}^{n}(z)$ are the Gegenbauer polynomials. The angles $0 \leq \Phi_{1} \leq 2 \pi$ and $0 \leq \Phi_{i} \leq \pi$ for $i \neq 1$ can be identified with the Euler angles of a rotation $g$ which maps the north pole $a=(0, \cdots, 0,1) \in \mathbb{R}^{d}$ to an arbitrary point on $S^{d-1}$. Then the hyperspherical harmonics on $S^{d-1}$ are defined as follows:

$$
|e\rangle=D^{l}(g)|a\rangle, \quad Y_{l \mathbf{M}}(e)=\langle e \mid l, \mathbf{M}\rangle, \quad\langle a \mid l, \mathbf{M}\rangle=\sqrt{\frac{d_{l}}{V_{d}}} \delta_{\mathbf{M} \mathbf{0}}
$$

where $V_{d}=\frac{2 \pi^{d / 2}}{\Gamma(d / 2)}$ is the volume of unit $S^{d-1}$ sphere. Therefore, we get

$$
Y_{l \mathbf{M}}(e)=\sqrt{\frac{d_{l}}{V_{d}}} D_{\mathbf{M} \mathbf{0}}^{l *}(g)
$$

We finally list the following properties of hyperspherical harmonics which can be easily derived using the definitions given above:

1. $Y_{l \mathbf{M}}^{*}(e)=(-1)^{m_{1}} Y_{l} \overline{\mathbf{M}}(e) \quad$ where $\quad \overline{\mathbf{M}}=\left(m_{d-2}, \cdots, m_{2},-m_{1}\right)$.

2. $Y_{l_{1} \mathbf{M}_{1}}(e) Y_{l_{2} \mathbf{M}_{2}}(e)=\sum_{l_{3}, \mathbf{M}_{3}}\left(\begin{array}{c}l_{1} l_{2} l_{3} \\ \mathbf{M}_{1} \mathbf{M}_{2} \mathbf{M}_{3}\end{array}\right)\left(\begin{array}{c}l_{1} l_{2} l_{3} \\ \mathbf{0 0 0}\end{array}\right) Y_{l_{3} \mathbf{M}_{3}}^{*}(e)$ 
3. $\left(\begin{array}{c}l_{1} l_{2} l_{3} \\ 000\end{array}\right)=0$ unless $l_{1}+l_{2}+l_{3}$ is an even integer and $l_{3}=\left|l_{1}-l_{2}\right|, \cdots, l_{1}+l_{2}$.

4. $\left(\begin{array}{c}l l^{\prime} 0 \\ \mathbf{M M}^{\prime} 0\end{array}\right)=\frac{(-1)^{l-m 1}}{\sqrt{d_{l}}} \delta_{l l^{\prime}} \delta_{\mathbf{M ~ M}^{\prime}}$

5. $\sum_{\left\{\mathbf{m}_{i}\right\}}(-1)^{\left(\mathbf{m}_{2}\right)_{1}}\left(\begin{array}{c}l_{1} l_{3} L_{2} \\ \mathbf{m}_{1} \mathbf{m}_{3} \mathbf{M}_{2}\end{array}\right)\left(\begin{array}{c}l_{1} l_{2} L_{3} \\ \overline{\mathbf{m}}_{1} \mathbf{m}_{2} \mathbf{M}_{3}\end{array}\right)\left(\begin{array}{c}l_{2} l_{3} L_{1} \\ \overline{\mathbf{m}}_{2} \overline{\mathbf{m}}_{3} \mathbf{M}_{1}\end{array}\right)$

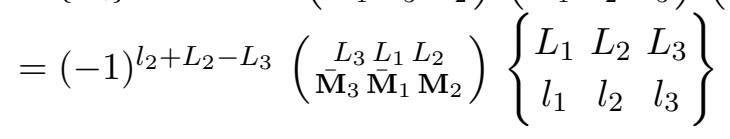

$\boldsymbol{s o}(\mathbf{1}, \boldsymbol{d}+\mathbf{1}) \mathbf{C G C}$ for scalar irreps. We extract $\mathrm{CG}$ coefficients for $s o(1, d+1)$ for three scalars from the three-point function for scalar primary operators amputating the out-going and in-going legs we had found earlier. The out-going and in-going legs takes the following forms respectively

$$
\begin{aligned}
\lim _{\rho \rightarrow \infty} e^{\rho \Delta}\langle\langle\Delta|g(x)| \Delta ;\{l, \mathbf{m}, s\}\rangle & =\frac{2^{l+2 s}}{A_{l, s}} \frac{\Gamma(\Delta+s+l) \Gamma(\Delta+s-\mu)}{\Gamma(\Delta) \Gamma(\Delta-\mu)} M_{\mathbf{m}}^{l}(\mathbf{x})\left(x^{2}\right)^{-\Delta-l-s} \\
& =\left[\frac{(\Delta)_{l+s}(\Delta-\mu)_{s}}{(\mu+1)_{l+s} s !}\right]^{1 / 2}\left(x^{2}\right)^{-\Delta-l-s} M_{\mathbf{m}}^{l}(\mathbf{x})
\end{aligned}
$$

and

$$
\begin{aligned}
\left.\lim _{\rho \rightarrow \infty} e^{\rho \Delta}\left\langle\Delta ;\{l, \mathbf{m}, s\}\left|g^{-1}(y)\right| \Delta\right\rangle\right\rangle & =\frac{A_{l, s}}{2^{l+2 s}} \frac{\left(y^{2}\right)^{s}}{(s) ! \Gamma(l+s+3 / 2)} M_{\mathbf{m}}^{l^{*}}(\mathbf{y}) \\
& =\left[\frac{(\Delta)_{l+s}(\Delta-\mu)_{s}}{(\mu+1)_{l+s} s !}\right]^{1 / 2}\left(y^{2}\right)^{s} M_{\mathbf{m}}^{l^{*}}(\mathbf{y})
\end{aligned}
$$

The 3-point function of the scalar primary operators with conformal dimensions $\Delta_{1}, \Delta_{2}$ and $\Delta_{3}$

$$
\frac{1}{\left|x_{2}-x_{1}\right|^{\Delta_{1}+\Delta_{2}-\Delta_{3}}\left|x_{3}-x_{2}\right|^{\Delta_{2}+\Delta_{3}-\Delta_{1}}\left|x_{3}-x_{1}\right|^{\Delta_{1}+\Delta_{3}-\Delta_{2}}}
$$

can be expanded as

$$
\begin{aligned}
\left(4 \pi^{d / 2}\right)^{3} & \prod_{i=1}^{3} \sum_{l_{i}=0}^{\infty} \sum_{s_{i}=0}^{\infty} \sum_{\mathbf{m}_{i}} \frac{\left(\Delta_{12} / 2\right)_{l_{1}+s_{1}}\left(\Delta_{12} / 2-\mu\right)_{s 1}}{(\mu+1)_{l_{1}+s_{1}} s_{1} !} \frac{\left(\Delta_{23} / 2\right)_{l_{2}+s_{2}}\left(\Delta_{23} / 2-\mu\right)_{s 2}}{(\mu+1)_{l_{2}+s_{2}} s_{2} !} \\
& \times \frac{\left(\Delta_{31} / 2\right)_{l_{3}+s_{3}}\left(\Delta_{31} / 2-\mu\right)_{s 3}}{(\mu+1)_{l_{3}+s_{3}} s_{3} !} \\
& \times\left(x^{2}\right)^{-\Delta_{1}-l_{1}-l_{3}-s_{1}-s_{3}}\left(y^{2}\right)^{-\Delta_{23} / 2-l_{2}+s_{1}-s_{2}}\left(z^{2}\right)^{s_{2}+s_{3}} \\
& \times M_{\mathbf{m}_{1}}^{l_{1}}(\mathbf{x}) M_{\mathbf{m}_{3}}^{l_{3}}(\mathbf{x}) M_{\mathbf{m}_{1}}^{l_{1}^{*}}(\mathbf{y}) M_{\mathbf{m}_{2}}^{l_{2}}(\mathbf{y}) M_{\mathbf{m}_{2}}^{l_{2}^{*}}(\mathbf{z}) M_{\mathbf{m}_{3}}^{l_{3}^{*}}(\mathbf{z})
\end{aligned}
$$

where

$$
\Delta_{12} \equiv \Delta_{1}+\Delta_{2}-\Delta_{3}, \quad \Delta_{23} \equiv \Delta_{2}+\Delta_{3}-\Delta_{1}, \quad \Delta_{31} \equiv \Delta_{3}+\Delta_{1}-\Delta_{2}
$$

We use the following identities:

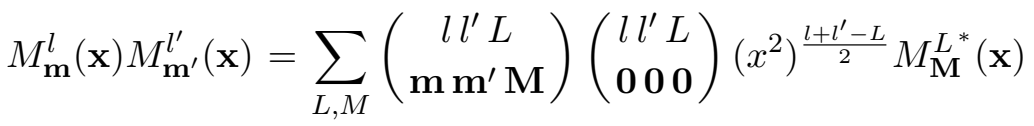

$$
\begin{aligned}
& M_{\mathbf{m}}^{l^{*}}(\mathbf{x})=(-1)^{m_{1}} M_{\bar{m}}^{l}(\mathbf{x})
\end{aligned}
$$


where, $\overline{\mathbf{m}}=\left(m_{n-2}, \ldots, m_{2},-m_{1}\right)$ to rewrite the product of spherical harmonics in the summand as

$$
\begin{aligned}
& M_{\mathbf{m}_{1}}^{l_{1}}(\mathbf{x}) M_{\mathbf{m}_{3}}^{l_{3}}(\mathbf{x}) M_{\mathbf{m}_{1}}^{l_{1}^{*}}(\mathbf{y}) M_{\mathbf{m}_{2}}^{l_{2}}(\mathbf{y}) M_{\mathbf{m}_{2}}^{l_{2}^{*}}(\mathbf{z}) M_{\mathbf{m}_{3}}^{l_{3}^{*}}(\mathbf{z}) \\
& =(-1)^{m_{1}+m_{2}+m_{3}} M_{\mathbf{m}_{1}}^{l_{1}}(\mathbf{x}) M_{\mathbf{m}_{3}}^{l_{3}}(\mathbf{x}) M_{\mathbf{m}_{1}}^{l_{1}}(\mathbf{y}) M_{\mathbf{m}_{2}}^{l_{2}}(\mathbf{y}) M_{\mathbf{m}_{2}}^{l_{2}}(\mathbf{z}) M_{\mathbf{m}_{3}}^{l_{3}}(\mathbf{z}) \\
& =(-1)^{m_{2}} \prod_{i=1}^{3} \sum_{L_{i}, M_{i}}(-1)^{M_{2}}\left(\begin{array}{ccc}
l_{1} & l_{3} & L_{2} \\
\mathbf{0} & 0 & 0
\end{array}\right)\left(\begin{array}{ccc}
l_{1} & l_{2} & L_{3} \\
\mathbf{0} & 0 & 0
\end{array}\right)\left(\begin{array}{ccc}
l_{2} & l_{3} & L_{1} \\
\mathbf{0} & 0 & 0
\end{array}\right) \\
& \times\left(\begin{array}{c}
l_{1} l_{3} L_{2} \\
\mathbf{m}_{1} \mathbf{m}_{3} \mathbf{M}_{2}
\end{array}\right)\left(\begin{array}{c}
l_{1} l_{2} L_{3} \\
\overline{\mathbf{m}}_{1} \mathbf{m}_{2} \mathbf{M}_{3}
\end{array}\right)\left(\begin{array}{c}
l_{2} l_{3} L_{1} \\
\overline{\mathbf{m}}_{2} \overline{\mathbf{m}}_{3} \mathbf{M}_{1}
\end{array}\right) \\
& \times\left(x^{2}\right)^{\frac{l_{1}+l_{3}-L_{2}}{2}}\left(y^{2}\right)^{\frac{l_{1}+l_{2}-L_{3}}{2}}\left(z^{2}\right)^{\frac{l_{2}+l_{3}-L_{1}}{2}} M_{\mathbf{M}_{2}}^{L_{2}}(\mathbf{x}) M_{\mathbf{M}_{3}}^{L_{3}}(\mathbf{y}) M_{\mathbf{M}_{1}}^{L_{1}}(\mathbf{z})
\end{aligned}
$$

Inserting the above relation in the summand and performing the $\left\{m_{i}\right\}$ summations we get

$$
\begin{gathered}
\sum_{\left\{\mathbf{m}_{i}\right\}}(-1)^{m_{2}}\left(\begin{array}{c}
l_{1} l_{3} L_{2} \\
\mathbf{m}_{1} \mathbf{m}_{3} \mathbf{M}_{2}
\end{array}\right)\left(\begin{array}{c}
l_{1} l_{2} L_{3} \\
\overline{\mathbf{m}}_{1} \mathbf{m}_{2} \mathbf{M}_{3}
\end{array}\right)\left(\begin{array}{c}
l_{2} l_{3} L_{1} \\
\overline{\mathbf{m}}_{2} \overline{\mathbf{m}}_{3} \mathbf{M}_{1}
\end{array}\right) \\
=(-1)^{l_{2}+L_{2}-L_{3}}\left(\begin{array}{c}
L_{3} L_{1} L_{2} \\
\overline{\mathbf{M}}_{3} \overline{\mathbf{M}}_{1} \mathbf{M}_{2}
\end{array}\right)\left\{\begin{array}{ccc}
l_{2} & l_{1} & L_{3} \\
L_{2} & L_{1} & l_{3}
\end{array}\right\} \\
=(-1)^{l_{2}+L_{2}-L_{3}}\left(\begin{array}{c}
L_{3} L_{1} L_{2} \\
\overline{\mathbf{M}}_{3} \overline{\mathbf{M}}_{1} \mathbf{M}_{2}
\end{array}\right)\left\{\begin{array}{ccc}
L_{1} & L_{2} & L_{3} \\
l_{1} & l_{2} & l_{3}
\end{array}\right\}
\end{gathered}
$$

Now the three-point function takes the form

$$
\begin{aligned}
& \left(4 \pi^{d / 2}\right)^{3} \prod_{i=1}^{3} \sum_{l_{i}=0}^{\infty} \sum_{s_{i}=0}^{\infty} \sum_{\mathbf{m}_{i}} \frac{\left(\Delta_{12} / 2\right)_{l_{1}+s_{1}}\left(\Delta_{12} / 2-\mu\right)_{s 1}}{(\mu+1)_{l_{1}+s_{1}} s_{1} !} \frac{\left(\Delta_{23} / 2\right)_{l_{2}+s_{2}}\left(\Delta_{23} / 2-\mu\right)_{s 2}}{(\mu+1)_{l_{2}+s_{2}} s_{2} !} \\
& \times \frac{\left(\Delta_{31} / 2\right)_{l_{3}+s_{3}}\left(\Delta_{31} / 2-\mu\right)_{s 3}}{(\mu+1)_{l_{3}+s_{3}} s_{3} !} \\
& \times \prod_{i=1}^{3} \sum_{L_{i}, \mathbf{M}_{i}}\left(x^{2}\right)^{-\Delta_{1}-l_{1}-l_{3}-s_{1}-s_{3}+\frac{l_{1}+l_{3}-L_{2}}{2}}\left(y^{2}\right)^{-\Delta_{23} / 2-l_{2}+s_{1}-s_{2}+\frac{l_{1}+l_{2}-L_{3}}{2}}\left(z^{2}\right)^{s_{2}+s_{3}+\frac{l_{2}+l_{3}-L_{1}}{2}} \\
& \times(-1)^{l_{2}+L_{2}-L_{3}+M_{2}} M_{\mathbf{M}_{2}}^{L_{2}}(\mathbf{x}) M_{\mathbf{M}_{3}}^{L_{3}}(\mathbf{y}) M_{\mathbf{M}_{1}}^{L_{1}}(\mathbf{z}) \\
& \times\left(\begin{array}{ccc}
l_{1} & l_{3} & L_{2} \\
\mathbf{0} & \mathbf{0} & \mathbf{0}
\end{array}\right)\left(\begin{array}{ccc}
l_{1} & l_{2} & L_{3} \\
\mathbf{0} & \mathbf{0} & \mathbf{0}
\end{array}\right)\left(\begin{array}{ccc}
l_{2} & l_{3} & L_{1} \\
\mathbf{0} & \mathbf{0} & \mathbf{0}
\end{array}\right)\left(\begin{array}{ccc}
L_{3} & L_{1} & L_{2} \\
\overline{\mathbf{M}}_{3} & \overline{\mathbf{M}}_{1} \mathbf{M}_{2}
\end{array}\right)\left\{\begin{array}{lll}
L_{1} & L_{2} & L_{3} \\
l_{1} & l_{2} & l_{3}
\end{array}\right\}
\end{aligned}
$$

which can also be written as

$$
\begin{aligned}
& \left(4 \pi^{d / 2}\right)^{3} \prod_{i=1}^{3} \sum_{l_{i}=0}^{\infty} \sum_{s_{i}=0}^{\infty} \sum_{\mathbf{m}_{i}} \frac{\left(\Delta_{12} / 2\right)_{l_{1}+s_{1}}\left(\Delta_{12} / 2-\mu\right)_{s 1}}{(\mu+1)_{l_{1}+s_{1}} s_{1} !} \\
& \times \frac{\left(\Delta_{23} / 2\right)_{l_{2}+s_{2}}\left(\Delta_{23} / 2-\mu\right)_{s 2}}{(\mu+1)_{l_{2}+s_{2}} s_{2} !} \frac{\left(\Delta_{31} / 2\right)_{l_{3}+s_{3}}\left(\Delta_{31} / 2-\mu\right)_{s 3}}{(\mu+1)_{l_{3}+s_{3}} s_{3} !} \\
& \times \prod_{i=1}^{3} \sum_{L_{i}, M_{i}}\left(x^{2}\right)^{-\Delta_{1}-l_{1}-l_{3}-s_{1}-s_{3}+\frac{l_{1}+l_{3}-L_{2}}{2}}\left(y^{2}\right)^{-\Delta_{23} / 2-l_{2}+s_{1}-s_{2}+\frac{l_{1}+l_{2}-L_{3}}{2}}\left(z^{2}\right)^{s_{2}+s_{3}+\frac{l_{2}+l_{3}-L_{1}}{2}} \\
& \times(-1)^{l_{2}+L_{2}-L_{3}} M_{\overline{\mathbf{M}}_{2}}^{L_{2}}(\mathbf{x}) M_{\overline{\mathbf{M}}_{3}}^{L_{3}^{*}}(\mathbf{y}) M_{\overline{\mathbf{M}}_{1}}^{L_{1}^{*}}(\mathbf{z}) \\
& \times\left(\begin{array}{ccc}
l_{1} & l_{3} & L_{2} \\
\mathbf{0} & 0 & 0
\end{array}\right)\left(\begin{array}{ccc}
l_{1} & l_{2} & L_{3} \\
\mathbf{0} & \mathbf{0} & \mathbf{0}
\end{array}\right)\left(\begin{array}{ccc}
l_{2} & l_{3} & L_{1} \\
\mathbf{0} & \mathbf{0} & \mathbf{0}
\end{array}\right)\left(\begin{array}{ccc}
L_{3} L_{1} L_{2} \\
\overline{\mathbf{M}}_{3} \overline{\mathbf{M}}_{1} \mathbf{M}_{2}
\end{array}\right)\left\{\begin{array}{ccc}
L_{1} & L_{2} & L_{3} \\
l_{1} & l_{2} & l_{3}
\end{array}\right\}
\end{aligned}
$$


Note that the $s o(d) 3 j$ - coefficients $\left(\begin{array}{c}l l^{\prime} L \\ 000\end{array}\right)$ is non-vanishing only when $l+l^{\prime}-L$ is even integer. This suggests to change the following variables as

$$
l_{1}+l_{3}=2 K_{2}+L_{2}, \quad l_{1}+l_{2}=2 K_{3}+L_{3}, \quad l_{2}+l_{3}=2 K_{1}+L_{1}
$$

i.e.

$$
\begin{aligned}
& l_{1}=\frac{L_{2}+L_{3}-L_{1}}{2}+K_{2}+K_{3}-K_{1} \\
& l_{2}=\frac{L_{3}+L_{1}-L_{2}}{2}+K_{3}+K_{1}-K_{2} \\
& l_{3}=\frac{L_{1}+L_{2}-L_{3}}{2}+K_{1}+K_{2}-K_{3}
\end{aligned}
$$

Then the powers of $x^{2}, y^{2}, z^{2}$ becomes (excluding the powers within the spherical harmonics)

$$
\left(x^{2}\right)^{-\Delta_{1}-L_{2}-K_{2}-s_{1}-s_{3}}\left(y^{2}\right)^{-\frac{\Delta_{23}}{2}-\frac{L_{3}+L_{1}-L_{2}}{2}+s_{1}-s_{2}-K_{1}+K_{2}}\left(z^{2}\right)^{s_{2}+s_{3}+K_{1}}
$$

respectively. Comparing with the legs we want to amputate from the three-point function we make the following change of variables in the summand

$$
\begin{aligned}
K_{2}+s_{1}+s_{3} & =S_{2} \\
K_{1}+s_{2}+s_{3} & =S_{1} \\
-\frac{\Delta_{23}}{2}-\frac{L_{3}+L_{1}-L_{2}}{2}+s_{1}-s_{2}-K_{1}+K_{2} & =S_{3}
\end{aligned}
$$

The last one of the above relations impose the following selection rule

$$
\left(\Delta_{2}+L_{1}+2 S_{1}\right)+\left(\Delta_{3}+L_{3}+2 S_{3}\right)=\left(\Delta_{1}+L_{2}+2 S_{2}\right)
$$

So $S_{3}$ is not an independent variable and $s_{3}$ is undetermined in terms of new variables. We call it $s_{3}=S$. In terms of the new variables the three-point function becomes

$$
\begin{aligned}
& \frac{\left(4 \pi^{d / 2}\right)^{3}}{\Gamma\left(\Delta_{12} / 2\right) \Gamma\left(\Delta_{23} / 2\right) \Gamma\left(\Delta_{31} / 2\right) \Gamma\left(\Delta_{12} / 2-\mu\right) \Gamma\left(\Delta_{23} / 2-\mu\right) \Gamma\left(\Delta_{31} / 2-\mu\right)} \\
& \times \prod_{i=1}^{3} \sum_{L_{i}=0}^{\infty} \sum_{S_{i}=0}^{\infty} \sum_{M_{i}} \delta\left(\Delta_{2}+L_{1}+2 S_{1}+\Delta_{3}+L_{3}+2 S_{3}-\Delta_{1}-L_{2}-2 S_{2}\right) \\
& \times \sum_{K_{3}=0}^{\infty} \sum_{K_{1}=0}^{S_{1}} \sum_{K_{2}=0}^{S_{2}} \sum_{S=0}^{\min \left(S_{2}-K_{2}, S_{1}-K_{1}\right)} \frac{\Gamma\left(\Delta_{2}+L_{3}+S_{1}+S_{3}+K_{3}-S-K_{1}\right) \Gamma\left(\Delta_{12} / 2+S_{2}-K_{2}-S-\mu\right)}{\Gamma\left(\frac{L_{2}+L_{3}-L_{1}}{2}+K_{3}-K_{1}+S_{2}-S+d / 2\right)\left(S_{2}-K_{2}-S\right) !} \\
& \times \frac{\Gamma\left(K_{3}-K_{2}+S_{2}-S_{3}-S\right) \Gamma\left(\Delta_{23} / 2+S_{1}-K_{1}-S-\mu\right)}{\Gamma\left(\frac{L_{3}+L_{1}-L_{2}}{2}+K_{3}-K_{2}+S_{1}-S+d / 2\right)\left(S_{1}-K_{1}-S\right) !} \\
& \times \frac{\Gamma\left(\Delta_{3}+L_{1}+\stackrel{2}{S}_{1}+S_{3}-S_{2}+K_{1}+K_{2}-K_{3}+S\right) \Gamma\left(\Delta_{31} / 2+S-\mu\right)}{\Gamma\left(\frac{L_{1}+L_{2}-L_{3}}{2}+K_{1}+K_{2}-K_{3}+S+d / 2\right) S !} \times(-1)^{\frac{L_{1}+L_{2}-L_{3}}{2}+K_{3}+K_{1}-K_{2}} \\
& \times\left(\begin{array}{cc}
\frac{L_{2}+L_{3}-L_{1}}{2}+K_{2}+K_{3}-K_{1}, \frac{L_{1}+L_{2}-L_{3}}{2}+K_{1}+K_{2}-K_{3}, L_{2} \\
0 & 0
\end{array}\right) \\
& \times\left(\begin{array}{cc}
\frac{L_{2}+L_{3}-L_{1}}{2}+K_{2}+K_{3}-K_{1}, \frac{L_{3}+L_{1}-L_{2}}{2}+K_{3}+K_{1}-K_{2}, L_{3} \\
0 & 0
\end{array}\right) \\
& \times\left(\begin{array}{cc}
\frac{L_{3}+L_{1}-L_{2}}{2}+K_{3}+K_{1}-K_{2}, \frac{L_{1}+L_{2}-L_{3}}{2}+K_{1}+K_{2}-K_{3}, L_{1} \\
0 & 0
\end{array}\right)
\end{aligned}
$$




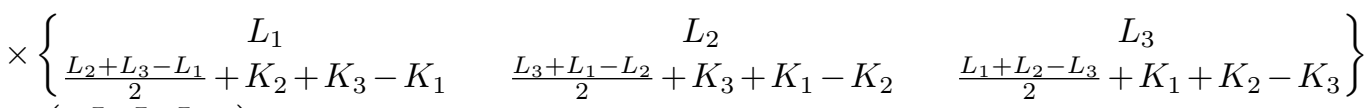

$$
\begin{aligned}
& \times\left(\begin{array}{c}
L_{3} L_{1} L_{2} \\
\mathbf{M}_{3} \mathbf{M}_{1} \mathbf{M}_{2}
\end{array}\right)\left(x^{2}\right)^{-\Delta_{1}-L_{2}-S_{2}}\left(y^{2}\right)^{S_{1}}\left(z^{2}\right)^{S_{3}} M_{-\mathbf{M}_{2}}^{L_{2}^{*}}(\mathbf{x}) M_{-\mathbf{M}_{3}}^{L_{3}}(\mathbf{y}) M_{-\mathbf{M}_{1}}^{L_{1}}(\mathbf{z})
\end{aligned}
$$

where we have arranged the order as well as the limits of the summations appropriately. According to our prescription the three-point function can be recovered as

$$
\begin{aligned}
\prod_{i=1}^{3} \sum_{L_{i}=0}^{\infty} \sum_{S_{i}=0}^{\infty} \sum_{\mathbf{M}_{i}} & \left\langle\left\langle\Delta_{1}|g(x)| \Delta_{1} ;\left\{L_{2}, \mathbf{M}_{2}, S_{2}\right\}\right\rangle\left\langle\Delta_{2} ;\left\{L_{1}, \mathbf{M}_{1}, S_{1}\right\}\left|g^{-1}(y)\right| \Delta_{2}\right\rangle\right\rangle \\
& \left.\times\left\langle\Delta_{3} ;\left\{L_{3}, \mathbf{M}_{3}, S_{3}\right\}\left|g^{-1}(z)\right| \Delta_{3}\right\rangle\right\rangle C_{\left(L_{1}, \mathbf{M}_{1}\right),\left(L_{3}, \mathbf{M}_{3}\right) ;\left(L_{2}, \mathbf{M}_{2}\right)}^{\left(\Delta_{2}, S_{1}\right),\left(\Delta_{3}, S_{3}\right) ;\left(\Delta_{1}, S_{2}\right)}
\end{aligned}
$$

where $C_{\left(L_{1}, \mathbf{M}_{1}\right),\left(L_{3}, \mathbf{M}_{3}\right) ;\left(L_{2}, \mathbf{M}_{2}\right)}^{\left(\Delta_{2}, S_{1}\right),\left(\Delta_{3}, S_{3}\right)\left(\Delta_{1}, S_{2}\right)}$ is $s o(1, d+1)$ CG coefficient. Comparing above with the three-point function we write

$$
\begin{aligned}
& C_{\left(L_{1}, \mathbf{M}_{1}\right),\left(L_{3}, \mathbf{M}_{3}\right) ;\left(L_{2}, \mathbf{M}_{2}\right)}^{\left(\Delta_{2}\right),\left(\Delta_{3}, S_{3}\right) ;\left(\Delta_{1}, S_{2}\right)} \\
& =\frac{\left(4 \pi^{d / 2}\right)^{3} \delta\left(\Delta_{2}+L_{1}+2 S_{1}+\Delta_{3}+L_{3}+2 S_{3}-\Delta_{1}-L_{2}-2 S_{2}\right)}{\Gamma\left(\Delta_{12} / 2\right) \Gamma\left(\Delta_{23} / 2\right) \Gamma\left(\Delta_{31} / 2\right) \Gamma\left(\Delta_{12} / 2-\mu\right) \Gamma\left(\Delta_{23} / 2-\mu\right) \Gamma\left(\Delta_{31} / 2-\mu\right)} \\
& \times\left[\frac{\Gamma\left(\Delta_{1}+L_{2}+S_{2}\right) \Gamma\left(\Delta_{1}+S_{2}-\mu\right)}{\Gamma\left(\Delta_{1}\right) \Gamma\left(\Delta_{1}-\mu\right) \Gamma\left(L_{2}+S_{2}+d / 2\right) S_{2} !}\right]^{1 / 2}\left[\frac{\Gamma\left(\Delta_{2}+L_{1}+S_{1}\right) \Gamma\left(\Delta_{2}+S_{1}-\mu\right)}{\Gamma\left(\Delta_{2}\right) \Gamma\left(\Delta_{2}-\mu\right) \Gamma\left(L_{1}+S_{1}+d / 2\right) S_{1} !}\right]^{1 / 2} \\
& \times\left[\frac{\Gamma\left(\Delta_{3}+L_{3}+S_{3}\right) \Gamma\left(\Delta_{3}+S_{3}-\mu\right)}{\Gamma\left(\Delta_{3}\right) \Gamma\left(\Delta_{3}-\mu\right) \Gamma\left(L_{3}+S_{3}+d / 2\right) S_{3} !}\right]^{1 / 2} \\
& \times \sum_{K_{3}=0}^{\infty} \sum_{K_{1}=0}^{S_{1}} \sum_{K_{2}=0}^{S_{2}} \sum_{S=0}^{\min \left(S_{2}-K_{2}, S_{1}-K_{1}\right)}(-1)^{\frac{L_{1}+L_{2}-L_{3}}{2}+K_{3}+K_{1}-K_{2}} \\
& \times \frac{\Gamma\left(\Delta_{2}+L_{3}+S_{1}+S_{3}+K_{3}-S-K_{1}\right) \Gamma\left(\Delta_{12} / 2+S_{2}-K_{2}-S-\mu\right)}{\Gamma\left(\frac{L_{2}+L_{3}-L_{1}}{2}+K_{3}-K_{1}+S_{2}-S+d / 2\right)\left(S_{2}-K_{2}-S\right) !} \\
& \times \frac{\Gamma\left(K_{3}-K_{2}+S_{2}-S_{3}-S\right) \Gamma\left(\Delta_{23} / 2+S_{1}-K_{1}-S-\mu\right)}{\Gamma\left(\frac{L_{3}+L_{1}-L_{2}}{2}+K_{3}-K_{2}+S_{1}-S+d / 2\right)\left(S_{1}-K_{1}-S\right) !} \\
& \times \frac{\Gamma\left(\Delta_{3}+L_{1}+S_{1}+S_{3}-S_{2}+K_{1}+K_{2}-K_{3}+S\right) \Gamma\left(\Delta_{31} / 2+S-\mu\right)}{\Gamma\left(\frac{L_{1}+L_{2}-L_{3}}{2}+K_{1}+K_{2}-K_{3}+S+d / 2\right) S !}
\end{aligned}
$$

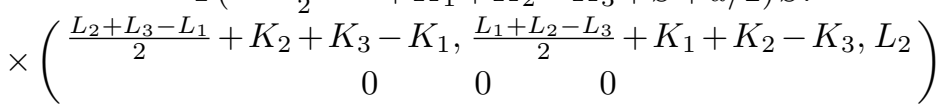

$$
\begin{aligned}
& \times\left(\begin{array}{ccc}
\frac{L_{2}+L_{3}-L_{1}}{2}+K_{2}+K_{3}-K_{1}, \frac{L_{3}+L_{1}-L_{2}}{2}+K_{3}+K_{1}-K_{2}, L_{3} \\
0 & 0 & 0
\end{array}\right)
\end{aligned}
$$

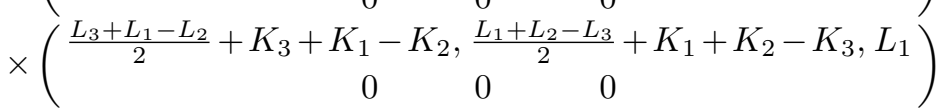

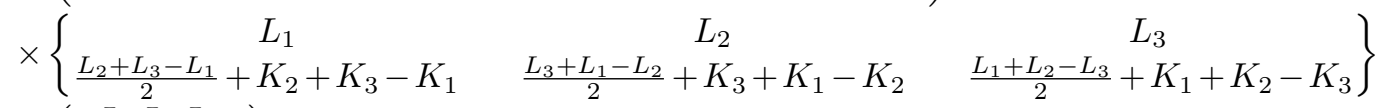

$$
\begin{aligned}
& \times\left(\begin{array}{c}
L_{3} L_{1} L_{2} \\
\mathbf{M}_{3} \mathbf{M}_{1} \mathbf{M}_{2}
\end{array}\right)
\end{aligned}
$$




\section{B Manipulation of the $d$-dimensional result}

The four-point block of a scalar correlation function in general dimensions in our method takes the following form:

$$
\begin{aligned}
\left(x^{2}\right)^{\frac{1}{2}\left(\Delta-\Delta_{3}-\Delta_{4}\right)} & \sum_{l, s} \Gamma(\alpha+l+s) \Gamma(\beta+l+s) \Gamma(\alpha+s-\mu) \Gamma(\beta+s-\mu) \\
& \times \frac{(l+\mu)}{s ! \Gamma(l+s+1+\mu) \Gamma(\Delta+l+s) \Gamma(\Delta+s-\mu)}\left(x^{2}\right)^{s} \mathcal{C}_{l}^{\mu}(\mathbf{x} \cdot \mathbf{u})
\end{aligned}
$$

where $\mu=\frac{d-2}{2}$. One of the questions we have to address is how our computations match with those known in the literature. There is a famous expression for the conformal blocks in any dimension in terms the cross ratios $u, v$ as found by Dolan and Osborn. We now prove the following identity towards establishing the equivalence between our answers and theirs.

$$
\sum_{l, s=0}^{\infty} \frac{\left(\frac{\Delta-\Delta_{12}}{2}\right)_{l+s}\left(\frac{\Delta+\Delta_{34}}{2}\right)_{l+s}}{(\Delta)_{l+s}} \frac{\left(\frac{\Delta-\Delta_{12}}{2}-\mu\right)_{s}\left(\frac{\Delta+\Delta_{34}}{2}-\mu\right)_{s}}{(\Delta-\mu)_{s}} \frac{1+\frac{l}{\mu}}{s !(\mu+1)_{l+s}}(z \bar{z})^{s+\frac{l}{2}} C_{l}^{\mu}\left(\frac{z+\bar{z}}{2 \sqrt{z \bar{z}}}\right)
$$

is equal to

$$
\sum_{r, q=0}^{\infty} \frac{\left(\frac{\Delta+\Delta_{12}}{2}\right)_{r}\left(\frac{\Delta-\Delta_{12}}{2}\right)_{r+q}\left(\frac{\Delta-\Delta_{34}}{2}\right)_{r}\left(\frac{\Delta+\Delta_{34}}{2}\right)_{r+q}}{r ! q !(\Delta)_{2 r+q}(\Delta-\mu)_{r}}(z \bar{z})^{r}(z+\bar{z}-z \bar{z})^{q}
$$

To establish this we first note the following identities/definitions:

$$
\begin{aligned}
(z \bar{z})^{\frac{l}{2}} C_{l}^{\mu}\left(\frac{z+\bar{z}}{2 \sqrt{z \bar{z}}}\right) & :=\sum_{k=0}^{[l / 2]}(-1)^{k} \frac{(\mu)_{l-k}}{k !(l-2 k) !}(z+\bar{z})^{l-2 k}(z \bar{z})^{k} \\
(z+\bar{z}-z \bar{z})^{q} & =\sum_{p=0}^{q}(-1)^{p}\left(\begin{array}{c}
q \\
p
\end{array}\right)(z+\bar{z})^{q-p}(z \bar{z})^{p}
\end{aligned}
$$

Using the double sum identity:

$$
\sum_{q=0}^{\infty} \sum_{p=0}^{q} a_{p, q-p}=\sum_{m=0}^{\infty} \sum_{n=0}^{\infty} a_{n, m}=\sum_{l=0}^{\infty} \sum_{k=0}^{[l / 2]} a_{k, l-2 k}
$$

the first expression can be written as

$$
\begin{aligned}
\sum_{s=0}^{\infty} & \frac{\left(\frac{\Delta-\Delta_{12}}{2}-\mu\right)_{s}\left(\frac{\Delta+\Delta_{34}}{2}-\mu\right)_{s}}{s !(\Delta-\mu)_{s}} \sum_{m, n=0}^{\infty} \frac{\left(\frac{\Delta-\Delta_{12}}{2}\right)_{s+m+2 n}\left(\frac{\Delta+\Delta_{34}}{2}\right)_{s+m+2 n}}{(\Delta)_{s+m+2 n} n ! m !} \\
& \times \frac{\left(1+\frac{m+2 n}{\mu}\right)(\mu)_{m+n}}{(\mu+1)_{s+m+2 n}}(-1)^{n}(z \bar{z})^{n+s}(z+\bar{z})^{m}
\end{aligned}
$$

The second of the expressions can be manipulated to:

$$
\sum_{r=0}^{\infty} \frac{\left(\frac{\Delta+\Delta_{12}}{2}\right)_{r}\left(\frac{\Delta-\Delta_{34}}{2}\right)_{r}}{r !(\Delta-\mu)_{r}} \sum_{m, p=0}^{\infty} \frac{\left(\frac{\Delta-\Delta_{12}}{2}\right)_{r+m+p}\left(\frac{\Delta+\Delta_{34}}{2}\right)_{r+m+p}}{m ! p !(\Delta)_{2 r+m+p}}(-1)^{p}(z \bar{z})^{r+p}(z+\bar{z})^{m}
$$


In the next step we extract the coefficients of $(z \bar{z})^{q}(z+\bar{z})^{m}$ in both these expressions. For this in the first expression we change $n \rightarrow p, s \rightarrow q-p$ and in the second we change $p \rightarrow p, r \rightarrow q-p$. Then in both the expressions the indices $q$ and $m$ run freely over all non-negative integers and the index $p$ runs over $0,1, \cdots, q$. The corresponding coefficient for the first expression is:

$$
\sum_{p=0}^{q} \frac{\left(\frac{\Delta-\Delta_{12}}{2}-\mu\right)_{q-p}\left(\frac{\Delta+\Delta_{34}}{2}-\mu\right)_{q-p}}{(q-p) !(\Delta-\mu)_{q-p}} \frac{\left(\frac{\Delta-\Delta_{12}}{2}\right)_{m+p+q}\left(\frac{\Delta+\Delta_{34}}{2}\right)_{m+p+q}}{(\Delta)_{m+p+q} p ! m !} \frac{\mu+m+2 p}{(\mu+m+p)_{q+1}}(-1)^{p}
$$

and for the second expression is:

$$
\sum_{p=0}^{q} \frac{\left(\frac{\Delta+\Delta_{12}}{2}\right)_{q-p}\left(\frac{\Delta-\Delta_{34}}{2}\right)_{q-p}}{(q-p) !(\Delta-\mu)_{q-p}} \frac{\left(\frac{\Delta-\Delta_{12}}{2}\right)_{m+q}\left(\frac{\Delta+\Delta_{34}}{2}\right)_{m+q}}{m ! p !(\Delta)_{m+2 q-p}}(-1)^{p}
$$

Now the final step is to compare these two expressions (B.9) and (B.10) for arbitrary integers $\{d \geq 1, q \geq 0, m \geq 0\}$. We conjecture that these expressions are identical. We have verified this claim for various special cases exactly, and for large subsets of the integer parameters $\{d \geq 1, q \geq 0, m \geq 0\}$ using Mathematica.

\section{Details of CPW computation in $d=4$}

To establish the result for four-point scalar CPW in $d=4$ we start by expanding the answer in power series.

$$
\begin{aligned}
z_{2} F_{1}(\alpha, \beta, \Delta, z){ }_{2} F_{1}(\alpha-1, \beta-1, \Delta-2, \bar{z})= & \sum_{m=0}^{\infty} \sum_{n=0}^{\infty} \frac{\Gamma(\alpha+m) \Gamma(\alpha+n-1)}{\Gamma(\alpha) \Gamma(\alpha-1)} \\
& \times \frac{\Gamma(\beta+m) \Gamma(\beta+n-1)}{\Gamma(\beta) \Gamma(\beta-1)} \\
& \times \frac{\Gamma(\Delta) \Gamma(\Delta-2)}{\Gamma(\Delta+m) \Gamma(\Delta+n-2)} \frac{z^{m+1} \bar{z}^{n}}{m ! n !}
\end{aligned}
$$

We now divide the r.h.s. into three terms with $m+1>n, m+1<n$ and $m+1=n$. The piece coming from terms with $m+1=n$ are real and therefore cancel with the corresponding terms from the complex conjugate combination. The remaining parts are obtained by considering the restricted sums

$$
\sum_{n=0}^{\infty} \sum_{m=n}^{\infty}+\sum_{m=0}^{\infty} \sum_{n=m+2}^{\infty}
$$

Let us consider the conjugate term next:

$$
\begin{aligned}
\bar{z}_{2} F_{1}(\alpha, \beta, \Delta, \bar{z}){ }_{2} F_{1}(\alpha-1, \beta-1, \Delta-2, z)= & \sum_{m=0}^{\infty} \sum_{n=0}^{\infty} \frac{\Gamma(\alpha+m) \Gamma(\alpha+n-1)}{\Gamma(\alpha) \Gamma(\alpha-1)} \\
& \times \frac{\Gamma(\beta+m) \Gamma(\beta+n-1)}{\Gamma(\beta) \Gamma(\beta-1)} \\
& \times \frac{\Gamma(\Delta) \Gamma(\Delta-2)}{\Gamma(\Delta+m) \Gamma(\Delta+n-2)} \frac{\bar{z}^{m+1} z^{n}}{m ! n !}
\end{aligned}
$$


again we split this into three types of terms as above and drop the term that is real. Then we can split the rest into two types of terms by writing the sum as before in two parts:

$$
\sum_{n=0}^{\infty} \sum_{m=n}^{\infty}+\sum_{m=0}^{\infty} \sum_{n=m+2}^{\infty}
$$

Noticing that the first sum in the first term and the second sum in the second have more $z$ 's than $\bar{z}$ 's we would like to combine them. In these two we introduce two new variables $m=n+p$ and $n=m+2+q$ to replace $m$ and $n$ respectively. Combing these we have:

$$
\begin{aligned}
\sum_{n=0}^{\infty} \sum_{p=0}^{\infty} & \frac{\Gamma(\alpha+n+p) \Gamma(\alpha+n-1)}{\Gamma(\alpha) \Gamma(\alpha-1)} \frac{\Gamma(\beta+n+p) \Gamma(\beta+n-1)}{\Gamma(\beta) \Gamma(\beta-1)} \\
& \times \frac{\Gamma(\Delta) \Gamma(\Delta-2)}{\Gamma(\Delta+n+p) \Gamma(\Delta+n-2)} \frac{z^{n+p+1} \bar{z}^{n}}{(n+p) ! n !} \\
-\sum_{m=0}^{\infty} \sum_{q=0}^{\infty} \frac{\Gamma(\alpha+m) \Gamma(\alpha+q+m+1)}{\Gamma(\alpha) \Gamma(\alpha-1)} \frac{\Gamma(\beta+m) \Gamma(\beta+q+m+1)}{\Gamma(\beta) \Gamma(\beta-1)} & \times \frac{\Gamma(\Delta) \Gamma(\Delta-2)}{\Gamma(\Delta+m) \Gamma(\Delta+q+m)} \frac{\bar{z}^{m+1} z^{q+m+2}}{m !(q+m+2) !}
\end{aligned}
$$

In the second term we can replace $m \rightarrow m-1$ and still sum over the new $m$ from 0 to $\infty$ as there will be term $(m-1)$ ! in the denominator which kills the $m=0$ term. Then

$$
\begin{aligned}
\sum_{n=0}^{\infty} \sum_{p=0}^{\infty} & \frac{\Gamma(\alpha+n+p) \Gamma(\alpha+n-1)}{\Gamma(\alpha) \Gamma(\alpha-1)} \frac{\Gamma(\beta+n+p) \Gamma(\beta+n-1)}{\Gamma(\beta) \Gamma(\beta-1)} \\
& \times \frac{\Gamma(\Delta) \Gamma(\Delta-2)}{\Gamma(\Delta+n+p) \Gamma(\Delta+n-2)} \frac{z^{n+p+1} \bar{z}^{n}}{(n+p) ! n !} \\
-\sum_{m=0}^{\infty} \sum_{q=0}^{\infty} & \frac{\Gamma(\alpha+m-1) \Gamma(\alpha+q+m)}{\Gamma(\alpha) \Gamma(\alpha-1)} \frac{\Gamma(\beta+m-1) \Gamma(\beta+q+m)}{\Gamma(\beta) \Gamma(\beta-1)} \\
& \times \frac{\Gamma(\Delta) \Gamma(\Delta-2)}{\Gamma(\Delta+m-1) \Gamma(\Delta+q+m-1)} \frac{\bar{z}^{m} z^{q+m+1}}{(m-1) !(q+m+1) !}
\end{aligned}
$$

Now we change dummy variables $n \rightarrow s, p \rightarrow l$ in the first term and $m \rightarrow s$ and $q \rightarrow l$ in the second term and combine terms to write this as:

$$
\begin{aligned}
\sum_{l=0}^{\infty} \sum_{s=0}^{\infty} \frac{}{} & \frac{}{(\alpha+l+s) \Gamma(\alpha+s-1)} \frac{\Gamma(\beta+l+s) \Gamma(\beta+s-1)}{\Gamma(\alpha) \Gamma(\alpha-1)} \frac{\Gamma(\beta-1)}{\Gamma(\Delta) \Gamma(\Delta-2)} \\
& \times \frac{z^{l+s+1} \bar{z}^{s}}{\Gamma(\Delta+s-2) \Gamma(\Delta+l+s-1)} \frac{1}{(s-1) !(l+s) !} \\
& \times\left[\frac{1}{(\Delta+l+s-1) s}-\frac{1}{(\Delta+s-2)(l+s+1)}\right]
\end{aligned}
$$

Using

$$
\frac{1}{(\Delta+l+s-1) s}-\frac{1}{(\Delta+s-2)(l+s+1)}=\frac{(\Delta-2)(l+1)}{(\Delta+l+s-1)(\Delta+s-2)(l+s+1) s}
$$


This can be seen to be:

$$
\begin{gathered}
\sum_{l=0}^{\infty} \sum_{s=0}^{\infty} \frac{\Gamma(\alpha+l+s) \Gamma(\alpha+s-1)}{\Gamma(\alpha) \Gamma(\alpha-1)} \frac{\Gamma(\beta+l+s) \Gamma(\beta+s-1)}{\Gamma(\beta) \Gamma(\beta-1)} \\
\times \frac{\Gamma(\Delta) \Gamma(\Delta-1)}{\Gamma(\Delta+s-1) \Gamma(\Delta+l+s)} \frac{z^{l+s+1} \bar{z}^{s}}{s !(l+s+1) !}
\end{gathered}
$$

which is precisely the first term in our OWN computation of the block. The remaining two terms are simply conjugates of what we have dealt with so far and therefore are going to reproduce the second term in our OWN computation.

Open Access. This article is distributed under the terms of the Creative Commons Attribution License (CC-BY 4.0), which permits any use, distribution and reproduction in any medium, provided the original author(s) and source are credited.

\section{References}

[1] F.A. Dolan and H. Osborn, Conformal partial waves: further mathematical results, arXiv:1108.6194 [INSPIRE].

[2] F.A. Dolan and H. Osborn, Conformal four point functions and the operator product expansion, Nucl. Phys. B 599 (2001) 459 [hep-th/0011040] [INSPIRE].

[3] F.A. Dolan and H. Osborn, Conformal partial waves and the operator product expansion, Nucl. Phys. B 678 (2004) 491 [hep-th/0309180] [inSPIRE].

[4] D. Simmons-Duffin, Projectors, shadows and conformal blocks, JHEP 04 (2014) 146 [arXiv: 1204.3894] [INSPIRE].

[5] S. El-Showk, M.F. Paulos, D. Poland, S. Rychkov, D. Simmons-Duffin and A. Vichi, Solving the 3D Ising model with the conformal bootstrap, Phys. Rev. D 86 (2012) 025022 [arXiv:1203.6064] [INSPIRE].

[6] M. Hogervorst, H. Osborn and S. Rychkov, Diagonal limit for conformal blocks in d dimensions, JHEP 08 (2013) 014 [arXiv: 1305.1321] [INSPIRE].

[7] M. Hogervorst and S. Rychkov, Radial coordinates for conformal blocks, Phys. Rev. D 87 (2013) 106004 [arXiv:1303.1111] [INSPIRE].

[8] E. Hijano, P. Kraus, E. Perlmutter and R. Snively, Witten diagrams revisited: the AdS geometry of conformal blocks, JHEP 01 (2016) 146 [arXiv: 1508.00501] [INSPIRE].

[9] E. Hijano, P. Kraus, E. Perlmutter and R. Snively, Semiclassical Virasoro blocks from AdS gravity, JHEP 12 (2015) 077 [arXiv:1508.04987] [INSPIRE].

[10] M. Nishida and K. Tamaoka, Geodesic Witten diagrams with an external spinning field, PTEP 2017 (2017) 053B06 [arXiv: 1609.04563] [INSPIRE].

[11] E. Dyer, D.Z. Freedman and J. Sully, Spinning geodesic Witten diagrams, JHEP 11 (2017) 060 [arXiv:1702.06139] [INSPIRE].

[12] H.-Y. Chen, E.-J. Kuo and H. Kyono, Anatomy of geodesic Witten diagrams, JHEP 05 (2017) 070 [arXiv: 1702.08818] [InSPIRE].

[13] A. Castro, E. Llabrés and F. Rejon-Barrera, Geodesic diagrams, gravitational interactions \& OPE structures, JHEP 06 (2017) 099 [arXiv: 1702.06128] [INSPIRE].

[14] V.A. Belavin and R.V. Geiko, Geodesic description of heavy-light Virasoro blocks, JHEP 08 (2017) 125 [arXiv:1705.10950] [INSPIRE]. 
[15] P. Kraus, A. Maloney, H. Maxfield, G.S. Ng and J.-Q. Wu, Witten diagrams for torus conformal blocks, JHEP 09 (2017) 149 [arXiv:1706.00047] [INSPIRE].

[16] K. Tamaoka, Geodesic Witten diagrams with antisymmetric tensor exchange, Phys. Rev. D 96 (2017) 086007 [arXiv:1707.07934] [INSPIRE].

[17] N. Anand, H. Chen, A.L. Fitzpatrick, J. Kaplan and D. Li, An exact operator that knows its location, JHEP 02 (2018) 012 [arXiv: 1708.04246] [INSPIRE].

[18] M. Nishida and K. Tamaoka, Fermions in geodesic Witten diagrams, JHEP 07 (2018) 149 [arXiv: 1805.00217] [INSPIRE].

[19] A. Bhatta, P. Raman and N.V. Suryanarayana, Holographic conformal partial waves as gravitational open Wilson networks, JHEP 06 (2016) 119 [arXiv: 1602.02962] [INSPIRE].

[20] M. Besken, A. Hegde, E. Hijano and P. Kraus, Holographic conformal blocks from interacting Wilson lines, JHEP 08 (2016) 099 [arXiv: 1603.07317] [INSPIRE].

[21] A.L. Fitzpatrick, J. Kaplan, D. Li and J. Wang, Exact Virasoro blocks from Wilson lines and background-independent operators, JHEP 07 (2017) 092 [arXiv: 1612.06385] [INSPIRE].

[22] M. Besken, A. Hegde and P. Kraus, Anomalous dimensions from quantum Wilson lines, arXiv: 1702.06640 [INSPIRE].

[23] Y. Hikida and T. Uetoko, Correlators in higher-spin AdS 3 holography from Wilson lines with loop corrections, PTEP 2017 (2017) 113B03 [arXiv: 1708.08657] [INSPIRE].

[24] Y. Hikida and T. Uetoko, Conformal blocks from Wilson lines with loop corrections, Phys. Rev. D 97 (2018) 086014 [arXiv:1801.08549] [INSPIRE].

[25] S.W. MacDowell and F. Mansouri, Unified geometric theory of gravity and supergravity, Phys. Rev. Lett. 38 (1977) 739 [Erratum ibid. 38 (1977) 1376] [INSPIRE].

[26] L. Freidel and A. Starodubtsev, Quantum gravity in terms of topological observables, hep-th/0501191 [INSPIRE].

[27] H. Verlinde, Poking holes in AdS/CFT: bulk fields from boundary states, arXiv: 1505.05069 [INSPIRE].

[28] M. Miyaji, T. Numasawa, N. Shiba, T. Takayanagi and K. Watanabe, Continuous multiscale entanglement renormalization ansatz as holographic surface-state correspondence, Phys. Rev. Lett. 115 (2015) 171602 [arXiv:1506.01353] [INSPIRE].

[29] Y. Nakayama and H. Ooguri, Bulk locality and boundary creating operators, JHEP 10 (2015) 114 [arXiv:1507.04130] [INSPIRE].

[30] B. Czech, L. Lamprou, S. McCandlish, B. Mosk and J. Sully, A stereoscopic look into the bulk, JHEP 07 (2016) 129 [arXiv: 1604.03110] [INSPIRE].

[31] J. de Boer, F.M. Haehl, M.P. Heller and R.C. Myers, Entanglement, holography and causal diamonds, JHEP 08 (2016) 162 [arXiv:1606.03307] [INSPIRE].

[32] A. Castro, N. Iqbal and E. Llabrés, Wilson lines and Ishibashi states in $A d S_{3} / C F T_{2}$, JHEP 09 (2018) 066 [arXiv: 1805.05398] [INSPIRE].

[33] G.A. Kerimov and I.A. Verdiev, Clebsch-Gordan coefficients of the groups $\mathrm{SO}(P, 1)$, Rept. Math. Phys. 20 (1984) 247 [inSPIRE].

[34] Z.-Y. Wen and J. Avery, Some properties of hyperspherical harmonics, J. Math. Phys. 26 (1985) 396.

[35] G. Junker, Explicit evaluation of coupling coefficients for the most degenerate representations of $\mathrm{SO}(n)$, J. Phys. A 26 (1993) 1649. 
[36] H.S. Cohl, On a generalization of the generating function for Gegenbauer polynomials, Integ. Trans. Spec. Funct. 24 (2013) 807.

[37] S. Terashima, AdS/CFT correspondence in operator formalism, JHEP 02 (2018) 019 [arXiv: 1710.07298] [INSPIRE].

[38] J. Penedones, E. Trevisani and M. Yamazaki, Recursion relations for conformal blocks, JHEP 09 (2016) 070 [arXiv: 1509.00428] [INSPIRE].

[39] M. Hogervorst, Dimensional reduction for conformal blocks, JHEP 09 (2016) 017 [arXiv: 1604.08913] [INSPIRE].

[40] J. Qiao and S. Rychkov, A tauberian theorem for the conformal bootstrap, JHEP 12 (2017) 119 [arXiv: 1709.00008] [INSPIRE].

[41] D.J. Gross and V. Rosenhaus, All point correlation functions in SYK, JHEP 12 (2017) 148 [arXiv: 1710.08113] [INSPIRE].

[42] R. Jackiw and V.P. Nair, Relativistic wave equations for anyons, Phys. Rev. D 43 (1991) 1933 [inSPIRE].

[43] M. Bañados, Three-dimensional quantum geometry and black holes, AIP Conf. Proc. 484 (1999) 147 [hep-th/9901148] [INSPIRE].

[44] S.S. Gubser, Absorption of photons and fermions by black holes in four-dimensions, Phys. Rev. D 56 (1997) 7854 [hep-th/9706100] [INSPIRE].

[45] S. Datta and J.R. David, Higher spin quasinormal modes and one-loop determinants in the BTZ black hole, JHEP 03 (2012) 079 [arXiv:1112.4619] [INSPIRE].

[46] B. Chen and J.-Q. Wu, Higher spin entanglement entropy at finite temperature with chemical potential, JHEP 07 (2016) 049 [arXiv: 1604.03644] [INSPIRE].

[47] A. Bhatta, P. Raman and N.V. Suryanarayana, Notes on general CPW from gravitational OWNs in $2 d$ CFTs, unpulished.

[48] A. Bhatta, P. Raman and N.V. Suryanarayana, Towards general holographic CPW in higher dimensional CFTs, work in progress.

[49] M.S. Costa, J. Penedones, D. Poland and S. Rychkov, Spinning conformal blocks, JHEP 11 (2011) 154 [arXiv:1109.6321] [InSPIRE].

[50] D. Karateev, P. Kravchuk and D. Simmons-Duffin, Weight shifting operators and conformal blocks, JHEP 02 (2018) 081 [arXiv: 1706. 07813] [INSPIRE].

[51] M.S. Costa and T. Hansen, AdS weight shifting operators, JHEP 09 (2018) 040 [arXiv: 1805.01492] [INSPIRE].

[52] Y. Nakayama, Bootstrapping critical Ising model on three-dimensional real projective space, Phys. Rev. Lett. 116 (2016) 141602 [arXiv:1601.06851] [InSPIRE].

[53] D.M. McAvity and H. Osborn, Conformal field theories near a boundary in general dimensions, Nucl. Phys. B 455 (1995) 522 [cond-mat/9505127] [INSPIRE].

[54] A. Gadde, Conformal constraints on defects, arXiv:1602.06354 [INSPIRE].

[55] A. Hamilton, D.N. Kabat, G. Lifschytz and D.A. Lowe, Local bulk operators in AdS/CFT: a holographic description of the black hole interior, Phys. Rev. D 75 (2007) 106001 [Erratum ibid. D 75 (2007) 129902] [hep-th/0612053] [INSPIRE]. 